\title{
Dark Matter in the Little Higgs Models
}

by

\section{Dmitriy Tseliakhovich}

\author{
A thesis submitted to the \\ Faculty of Graduate Studies and Research \\ in partial fulfillment of the requirements \\ for the degree of \\ Master of Science \\ Department of Physics \\ Carleton University \\ Ottawa-Carleton Institute of Physics \\ Ottawa, Canada
}

August 28, 2008

Copyright (c) 2008 Dmitriy Tseliakhovich 


$\begin{array}{ll}\begin{array}{l}\text { Library and } \\ \text { Archives Canada }\end{array} & \begin{array}{l}\text { Bibliothèque et } \\ \text { Archives Canada }\end{array} \\ \begin{array}{l}\text { Published Heritage } \\ \text { Branch }\end{array} & \begin{array}{l}\text { Direction du } \\ \text { Patrimoine de l'édition }\end{array} \\ \begin{array}{l}\text { 395 Wellington Street } \\ \text { Ottawa ON K1A 0N4 } \\ \text { Canada }\end{array} & \begin{array}{l}\text { 395, rue Wellington } \\ \text { Ottawa ON K1A 0N4 } \\ \text { Canada }\end{array}\end{array}$

Your file Votre référence ISBN: 978-0-494-44145-9 Our file Notre référence ISBN: 978-0-494-44145-9

NOTICE:

The author has granted a nonexclusive license allowing Library and Archives Canada to reproduce, publish, archive, preserve, conserve, communicate to the public by telecommunication or on the Internet, loan, distribute and sell theses worldwide, for commercial or noncommercial purposes, in microform, paper, electronic and/or any other formats.

The author retains copyright ownership and moral rights in this thesis. Neither the thesis nor substantial extracts from it may be printed or otherwise reproduced without the author's permission.
AVIS:

L'auteur a accordé une licence non exclusive permettant à la Bibliothèque et Archives Canada de reproduire, publier, archiver, sauvegarder, conserver, transmettre au public par télécommunication ou par l'Internet, prêter, distribuer et vendre des thèses partout dans le monde, à des fins commerciales ou autres, sur support microforme, papier, électronique et/ou autres formats.

L'auteur conserve la propriété du droit d'auteur et des droits moraux qui protège cette thèse. $\mathrm{Ni}$ la thèse ni des extraits substantiels de celle-ci ne doivent être imprimés ou autrement reproduits sans son autorisation.
In compliance with the Canadian Privacy Act some supporting forms may have been removed from this thesis.

While these forms may be included in the document page count, their removal does not represent any loss of content from the thesis.
Conformément à la loi canadienne sur la protection de la vie privée, quelques formulaires secondaires ont été enlevés de cette thèse.

Bien que ces formulaires aient inclus dans la pagination, il n'y aura aucun contenu manquant.

\section{Canada}




\section{Abstract}

Extensive astronomical evidence indicates that most of the matter in the universe is in the form of weakly-interacting massive particles (WIMPs). The Standard Model (SM) of particle physics, however, does not offer any candidate to account for the WIMP. Remarkably, many extensions of the SM, like supersymmetry, KleinKaluza models, or Little Higgs models, introduce new particles, some of which are excellent dark matter candidates. We investigate dark matter candidates that arise in the Little Higgs models after the introduction of a discrete symmetry (analogous to the R-parity of SUSY) called T-parity. The viability of Little Higgs dark matter is examined using the standard relic abundance constraints. We analyze collider signatures of dark matter candidates in the Simplest Little Higgs and Littlest Higgs with T-parity models. We investigate the impact of microscopic properties of dark matter on structure formation and discuss the prospects for future astrophysical studies that are needed to better understand the properties of dark matter. 
Per Aspera Ad Astra 


\section{Acknowledgments}

First, I would like to thank my adviser professor Pat Kalyniak for taking me as a student, the advice and guidance throughout this research, and for enabling the life-long experience that I have obtained at Carleton University.

I am mostly indebted to Dr. Mario Livio for inviting me to the Space Telescope Science Institute and for opening the world of cosmology for me. Our discussions and talks are the most enlightening and encouraging experience of my life.

I owe much gratitude to Dr. Michael Santos for his supervision and inestimable support during my stay at the STScI. Our cooperation was the brightest scientific experience a person may dream of.

I would like to thank my friend Aliaksei Charnukha from the University of Stutgart for the prolific discussions and for his help with the technical problems encountered during various stages of the project.

I also would like to thank Dr. Avi Loeb and his group for useful discussions of the structure formation part of this study and for the hospitality during my visit to the Harvard-Smithsonian Center for Astrophysics.

Most importantly, I would like to thank my family for their love and support throughout my life. I am especially grateful to my mother for her advice and encouragement in all my endeavors.

Finely, I would like to thank all those people whose names were not mentioned above, but whose contribution and advise have found their way into the results presented in this thesis. I am mostly grateful for your help and support. 


\section{Statement of Originality}

Included in this thesis is a review of the author's work resulting from research as a M.Sc. student at Carleton University and as a visiting research scholar at the Space Telescope Science Institute (STScI). The results within are not yet published, though several papers on the analysis performed are forthcoming.

The results contained within this thesis are entirely the work of the author, under the supervision of Dr. Pat Kalyniak at Carleton University and Dr. Michael Santos at the STScI. The review topics within the introductory chapters contain information obtained from other sources, and have been properly referenced and attributed as such. 


\section{Contents}

1 Introduction 1

1.1 The Standard Model of particle physics and its problems . . . . . . 4

1.2 Dark Matter in the Universe . . . . . . . . . . . . . 7

1.2.1 Astrophysical evidence for dark matter . . . . . . . . . . 8

1.2.2 Relic abundance of dark matter . . . . . . . . . 10

2 Dark matter in Little Higgs models 15

2.1 Littlest Higgs with T-parity . . . . . . . . . . . . . 16

2.2 Simplest Little Higgs with T-parity . . . . . . . . . . . . 20

3 Dark matter properties and the formation of complex structures in $\begin{array}{ll}\text { the Universe } & 29\end{array}$

3.1 Formalism of structure formation . . . . . . . . . . 30

3.2 Transfer function and prospective research . . . . . . . . . . 33

4 Collider phenomenology $\quad 36$ 
4.1 Dark matter and missing transverse energy . . . . . . . . . . . . . 37

4.2 MadGraph and calculation methods . . . . . . . . . . . . 40

4.3 LHC signatures of Little Higgs dark matter . . . . . . . . . . . . . 42

5 Results $\quad 50$

6 Summary $\quad 65$

$\begin{array}{ll}\text { Appendices } & 67\end{array}$

A Feynman Rules for the Simplest Little Higgs with T-parity model . . 67

B Parameters of the Simplest Little Higgs with T-parity model . . . . . 72 


\section{List of Tables}

$1.1 S U(3)_{c} \otimes S U(2)_{L} \otimes U(1)_{Y}$ representations of the SM fermions. The index $m$ runs over 3 generations of quarks and leptons. Subindices $\mathrm{L}$ and $\mathrm{R}$ distinguish left-handed and right-handed fields respectively. The subscript Y stands for the weak hypercharge. . . . . . . . . .

2.1 The $S U(3)_{c} \otimes S U(3)_{W} \otimes U(1)_{X}$ representations of the fermions in the anomaly-free embedding of the Simplest Little Higgs with T-parity model. Index a runs over the first two generations of quarks, while $m$ runs over all three generations of leptons. . . . . . . . . . . .

5.1 Parameters used for the analysis of dark matter production at the LHC in the Simplest Little Higgs with T-parity model. Values of the tested parameters are chosen to be consistent with the relic density constraints from Fig. 5.1 and to cover most of the allowed parameter space 
A1 Feynman rules for the triple gauge boson interactions in the Simplest Little Higgs with T-parity model. All momenta are defined as outgoing. The SM couplings of $W^{ \pm}$to $Z^{0}$ and photon are also presented for reference. In the table $s_{W}, c_{W}$, and $t_{W}$ stand for the sine, cosine and tangent of the electroweak mixing angle respectively. . . . . . . .

A2 Feynman rules for the quartic gauge boson interactions in the Simplest Little Higgs with T-parity model. Sample SM couplings are also provided for reference. . . . . . . . . . . . .

A3 Feynman rules for scalar-fermion couplings in the Simplest Little Higgs with T-parity model. $P_{L}=\left(1-\gamma^{5}\right) / 2$ is a projection operator. Only the leading terms are provided. . . . . . . . . . . .

A4 Feynman rules for gauge boson-fermion couplings in the Simplest Little Higgs with T-parity model. $P_{L}=\left(1-\gamma^{5}\right) / 2$ and $P_{R}=\left(1+\gamma^{5}\right) / 2$ are projection operators. Couplings for the second generation quarks are identical to those of the first generation. The SM lepton $Z^{0}$-boson coupling is also provided to clarify our phase choice. Only the leading terms are provided. . . . . . . . . . . . . .

A5 Feynman rules for gauge boson-scalar couplings in the Simplest Little Higgs with T-parity model. The SM coupling of the $W^{ \pm}$to the Higgs field $h$ is also provided for reference. Factor of $g_{\mu \nu}$ is implied in each

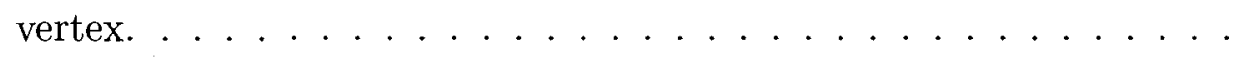




\section{List of Figures}

1.1 Rotation curve of the spiral galaxy NGC 6503. The dotted, dashed and dash-dotted lines are the contributions of gas, disk and dark matter, respectively. Figure adopted from Ref. [26]. . . . . . . . . . . .

2.1 The leading annihilation processes which maintain the heavy photon of the Littlest Higgs with T-parity model in equilibrium with the rest of the cosmic plasma in the early universe. . . . . . . . . . .

2.2 Dominant channels for dark matter annihilation in the Simplest Little Higgs with T-parity model. These diagrams provide the largest contributions to the heavy neutrino annihilation cross section for the range of SLHT parameters examined in this thesis. . . . . . . . . . .

2.3 t-channel contribution (short-dash line) to the thermally averaged annihilation cross section $\langle\sigma v\rangle$ (dashed line) in the Simplest Little Higgs with T-parity model as a function of dark matter mass. The WMAP allowed region [2] (gray) is also shown for reference. This plot emphasizes the contribution of the t-channel diagrams to the annihilation cross section. . . . . . . . . . . . . . . . 
3.1 Dominant channels for the Littlest Higgs with T-parity dark matter scattering with SM particles in the primordial plasma. This scattering maintains dark matter in kinetic equilibrium with the surrounding cosmic plasma long after freeze-out and determines the formation of the smallest dark matter halos. . . . . . . . . . . . .

3.2 Transfer function for the Littlest Higgs dark matter perturbations after the period of kinetic decoupling. The solid (dotted) line corresponds to $M_{A_{h}}=100 \mathrm{GeV}\left(M_{A_{h}}=300 \mathrm{GeV}\right)$. Other model parameters are chosen to be consistent with relic density constraints. The dashed line shows the transfer function in the CDM cosmology [19]. . . . . . .

4.1 Sample Feynman diagrams for $p p \rightarrow W_{h}^{+} W_{h}^{-} \rightarrow W^{+} W^{-} A_{h} A_{h}$ in the Littlest Higgs with T-parity model (left) and SM background (right).

4.2 Sample Feynman diagrams for $p p \rightarrow W_{h}^{ \pm} Z_{h} \rightarrow W^{ \pm} h A_{h} A_{h}$ in the Littlest Higgs with T-parity model (left) and SM background (right). .

4.3 Sample Feynman diagrams for the $\mathrm{W}$ associated dark matter production at the LHC in the Littlest Higgs with T-parity model (left) and the SM background for this process (right) . . . . . . . . . . . . . 46

4.4 Sample Feynman diagrams for the monojet associated dark matter production at the LHC in the Simplest Little Higgs with T-parity model. 47

4.5 Sample Feynman diagrams for the dileptonic dark matter production at the LHC in the Simplest Little Higgs with T-parity model. . . . 
4.6 Sample Feynman diagrams for the Standard Model background to the dileptonic (left) and monojet (right) associated dark matter production at the LHC. . . . . . . . . . . . . . . . . .

4.7 Cross section of dark matter production at the LHC is plotted as a function of dark matter mass. On the top scale we identify the SLHT symmetry breaking scale $f$ that corresponds to the dark matter mass via the relic density constraints of Eq. 5.1. The number of events for an integrated luminosity of $300 \mathrm{fb}^{-1}$ is plotted on the second y-axis. .

4.8 Cross section for dark matter production at the Tevatron in the Simplest Little Higgs Model. On the top scale we identify the SLHT symmetry breaking scale $f$ that corresponds to the dark matter mass via the relic density constraints of Eq. 5.1. The number of events for 8

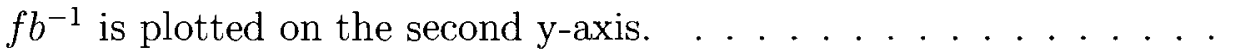

5.1 Relic abundance constraints on the parameter space of the Simplest Little Higgs with T-parity model. On this plot the dark region corresponds to $2 \sigma$ variation about the WMAP observed relic density, while the gray region covers $5 \sigma$ confidence level. . . . . . . . . . . .

5.2 Missing transverse energy distribution for the monojet plus missing energy production in the Simplest Little Higgs with T-parity model at the LHC and the SM background. This plot was generated with MadGraph using set 1 model parameters of Table 5, for the integrated luminosity of $300 \mathrm{fb}^{-1} \ldots \ldots \ldots \ldots$ 
5.3 Missing transverse energy distribution for the dilepton plus missing energy production in the Simplest Little Higgs with T-parity model at the LHC and the SM background. This plot was generated with MadGraph using set 1 model parameters of Table 5, for the integrated luminosity of $10 \mathrm{fb}^{-1} \ldots \ldots \ldots \ldots$

5.4 Comparison of the missing transverse energy distribution for the monojet associated dark matter production in the SLHT model at the LHC for the 3 parameter sets of Table 5 . The integrated luminosity is $300 \mathrm{fb}^{-1}$. Each distribution clearly exhibits a peak around the corresponding mass of $Z_{h} \ldots \ldots \ldots \ldots$

5.5 Comparison of the missing transverse energy distribution for the dilepton associated dark matter production in the SLHT model at the LHC for the 3 parameter sets of Table 5 . The integrated luminosity is $300 \mathrm{fb}^{-1} .58$

5.6 Expected $p_{T}$ distributions for the electrons in $p p \rightarrow l^{+} l^{-}+D M$ processes in the SLHT model for the first two parameter sets of Table 5. The SM $p_{T}$ distribution of electrons produced in $p p \rightarrow l^{+} l^{-}+$ Missing Energy processes is also plotted for comparison. $l^{+} l^{-}$represents either $e^{+} e^{-}$or $\mu^{+} \mu^{-}$pair. All curves are normalized by their total cross sections. . . . . . . . . . . . . . . 59 
5.7 Rapidity distributions for the observed electrons in $p p \rightarrow l^{+} l^{-}+D M$ processes in the SLHT model for the first two parameter sets of Table 5. The SM model rapidity distribution of electrons produced in $p p \rightarrow$ $l^{+} l^{-}+$Missing Energy processes is also presented. $l^{+} l^{-}$represents either $e^{+} e^{-}$or $\mu^{+} \mu^{-}$pair. All curves are normalized by their total

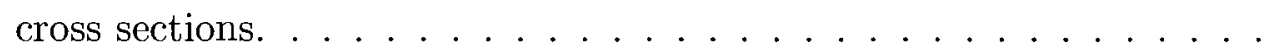

5.8 Invariant mass distributions of $e^{-}$and $e^{+}$in $p p \rightarrow l^{+} l^{-}+D M$ processes in the SLHT model for the first two parameter sets of Table 5. The SM model $m_{e^{+} e^{-}}$distribution produced in $p p \rightarrow l^{+} l^{-}+$Missing Energy processes is also presented. $l^{+} l^{-}$represents either $e^{+} e^{-}$or $\mu^{+} \mu^{-}$pair. All curves are normalized by their total cross sections. . . . . . . . .

5.9 Missing transverse energy distribution in dilepton plus missing energy production for the parameter set 2 of Table 5 with $p_{T}>200 \mathrm{GeV}$ cut imposed(left) and without cuts (right). SM expectation for this channel is also presented. . . . . . . . . . . . . .

5.10 Invariant mass distribution of electrons in dilepton plus missing energy production for the second parameter set of Table 5 with $p_{T}>200 \mathrm{GeV}$ cut imposed(left) and without cuts (right). SM expectation for this channel is also presented. . . . . . . . . . . . . . . . 
5.11 Expected missing transverse energy distribution for the $W^{ \pm}$associated dark matter production at the LHC in the Littlest Higgs with T-parity model for the dark matter mass $M_{A_{h}}=110 \mathrm{GeV}$ and symmetry breaking scale $f=700 \mathrm{GeV}$. The Higgs boson mass is chosen to be consistent with the relic density constraints of Eq. 2.8. This plot was generated using MadGraph for the integrated luminosity of $300 \mathrm{fb}^{-1}$. . . . .

5.12 Missing transverse energy distribution for $p p \rightarrow A_{h} A_{h} W^{+} W^{-}$in the Littlest Higgs with T-parity model at the LHC and the SM background. This plot was generated with MadGraph using $M_{A_{h}}=110 \mathrm{GeV}$ and symmetry breaking scale $f=700 \mathrm{GeV}$, for the integrated luminosity of $300 \mathrm{fb}^{-1} \ldots \ldots \ldots \ldots \ldots \ldots \ldots \ldots \ldots$

B1 $Z_{h}$ decay branching ratios in the Simplest Little Higgs with T-parity model for $f=1560 \mathrm{GeV}, M_{Z_{h}}=872 \mathrm{GeV}$, and $M_{h}=120 \mathrm{GeV}$. The solid, dotted, dashed and dash-dotted lines are the contributions of the dark matter, quark, neutrino, and $Z_{h} \rightarrow Z^{0}+h$ decay channels respectively. The total decay width of the heavy $Z_{h}$ boson is $\Gamma=5.8$ $\mathrm{GeV} \ldots \ldots \ldots \ldots \ldots \ldots \ldots \ldots$ 


\section{Chapter 1}

\section{Introduction}

The combined effort of theoretical and experimental research in physics and astronomy has resulted in the formulation of two extremely successful models that describe most of the observed phenomena to date. The current knowledge in particle physics is summarized in what is known as the Standard Model [1] while the results of the astrophysical observations are described within the Cold Dark Matter with dark energy $(\Lambda \mathrm{CDM})$ paradigm $[2,3]$. There is, however, almost universal acceptance that both models are incomplete. One particular problem inherent to both models, namely the inability to coherently explain the phenomenon of dark matter, has become the prime motivation of the study presented in this thesis.

Dark matter dominates the mass budget of the universe [2], but the actual particle(s) that comprise dark matter are still unknown. Dark matter has yet to be directly detected experimentally, despite attempts to intercept dark matter passing through Earth and attempts to generate dark matter with particle collisions. The broadest constraints on the properties of dark matter result from astronomical observations, which over the previous decades have supported what is known as the Cold 
Dark Matter (CDM) paradigm [4]. CDM is a phenomenological model, specifying a perfectly "cold" (i.e., without initial random motions) particle that interacts only through gravity, and is consequently also dissipationless. There are no astronomical observations or particle physics experiments that rule out a cold, non-interacting dark matter particle, and many observations that support it. However, some theoretical predictions based on CDM models are mildly at odds with observations probing the kpc-scale distribution of dark matter (e.g., [5]).

While the Standard Model (SM) of particle physics does not provide a natural non-baryonic dark matter candidate, the near-universal acceptance of dark matter as a major constituent of the universe has inspired many extensions to the Standard Model that, in addition to remedying other Standard Model deficiencies, provide a candidate dark matter particle. It is very important to note that no particle physics model produces a dark matter candidate conforming to the ideal of the CDM paradigm. Specifically, no particle can be perfectly cold or perfectly non-interacting. All dark matter particle models will predict some combination of random motions and non-gravitational interactions. Astronomical observations thus may move beyond providing a general confirmation of the CDM paradigm to specifically constraining particle physics models based on the dark matter properties they predict.

There is an exciting opportunity to combine theoretical models with upcoming dark matter direct and indirect detection experiments, results from new particle colliders like the Large Hadron Collider (LHC) at CERN, and astronomical observations, to finally assign an identity to the dark matter particle. To adequately address the opportunities opened by the upcoming experimental facilities it is imperative, from the theoretical side, to offer a broad variety of viable models with specific observational predictions. In this thesis we consider two realizations of the Little Higgs 
mechanism [6-11] - Simplest Little Higgs with T-parity (SLHT) [12] and Littlest Higgs with T-parity (LHT) [13-16] models.

Multiple astronomical observations constrain dark matter. The simplest constraint is on the amount of dark matter in the universe. Recent observations have improved measurements of the total mass density and baryonic mass density to the point that we now know the cosmic dark matter mass density to a precision of $10 \%$ [2]. Observations of high-energy radiation from locations of expected high dark-matter density (such as the centers of galaxies) place limits on the annihilation cross-section of dark matter with itself. The high precision of the current astrophysical searches provides an excellent possibility to constrain particle physics models. In the present study we focus on the relic density constraints that provide the most significant reduction of the allowed parameter space of any extension of the SM.

More complex and powerful constraints on the detailed nature of the dark matter particle arise from measurements of the spatial distribution of dark matter in the universe. Specifically, small deviations of the dark matter particle properties from the ideal CDM paradigm affect the small-scale spatial distribution of dark matter, precisely the regime where discrepancies between CDM-based model predictions and observations have been noted (e.g., [17-20]).

Imposing astrophysical constraints leads to a set of specific predictions for the observation and analysis of dark matter at the Large Hadron Collider at CERN. We provide various collider signatures of the Little Higgs dark matter and discuss prospects and difficulties for dark matter research at present day collider facilities.

Most of our discussion is centered around the Simplest Little Higgs with Tparity model [12] which has received very little attention in the literature so far. 
Also, our studies of structure formation are performed in a model independent way and potentially can find a broader application.

The rest of the thesis is organized as follows. In Section 1.1 we give a brief introduction to the SM and discuss its features and problems relevant to our research. Section 1.2 is devoted to the astrophysical evidence for dark matter. We review the key observations and discuss theoretical reasons that lead to the cold dark matter paradigm. Further, we show standard relic density calculation techniques that allow the imposition of stringent astrophysical constraints on the particle physics models. In Chapter 2 we review foundations of the Simplest and Littlest Higgs with T-parity models and discuss dark matter candidates that arise in those models. In Chapter 3 we discuss the extension of the $\Lambda$ CDM paradigm that accounts for the interaction of dark matter particles with the rest of the cosmic plasma after freeze-out and we illustrate specific predictions of such an extension. Collider phenomenology of the Little Higgs dark matter is discussed in Chapter 4. We outline the results of the relic density calculations as well as the results of the collider simulations in Chapter 5. A summary of our research follows in Chapter 6. Finally, we provide Feynman rules for the Simplest Little Higgs with T-parity model and discuss our choice of the model's parameters in the Appendices A and B respectively.

\subsection{The Standard Model of particle physics and its problems}

The Standard Model of particle physics [1] (for a review see for example [21]) is a quantum field theory that unifies electroweak theory and quantum chromodynamics. 
Its foundation is a local gauge symmetry known as $S U(3)_{c} \otimes S U(2)_{L} \otimes U(1)_{Y}$, spontaneously broken to $S U(3)_{c} \otimes U(1)_{e m}$ via the Higgs mechanism [22] (for a review see for example [23]). The $S U(3)_{c}$ is a symmetry of strong interactions and describes the interaction between particles that have color charge, while $S U(2)_{L} \otimes U(1)_{Y}$ describes the unified theory of electromagnetic and weak interactions. All matter particles are described by fundamental fields that transform under certain representations of the corresponding gauge groups.

Particles in the Standard Model can be divided into two classes based on their spin: bosons ( $\gamma, W^{ \pm}, Z^{0}$, gluons, and the Higgs boson) have integer spin and obey Bose-Einstein statistics, while fermions (three generations of quarks and leptons) have half-integer spin and satisfy Fermi-Dirac statistics. $W^{ \pm}, Z^{0}, \gamma$, and gluons are the mass eigenstates of the so-called gauge bosons that arise in the theory when local invariance under the corresponding gauge group is imposed and spontaneously broken. Gauge bosons transform under the adjoint representation of their gauge groups. In addition, particles are characterized by the direction of their spin relative to the direction of their motion as it is viewed by an inertial reference frame observer. A particle with its spin oriented along (opposite) its direction of motion is called right-handed (left-handed). Representations of the fundamental fermion fields and hypercharges in the SM are given in Table 1.1. Hypercharge $Y$ is related to the electrical charge $Q_{e m}$ of a particle and the third component of its weak isospin $T_{3}$ as

$$
Y=2\left(Q_{e m}-T_{3}\right)
$$

The invariance of the electroweak lagrangian under the local $S U(2)_{L} \otimes U(1)_{Y}$ gauge symmetry prohibits the appearance of mass terms. The generation of masses in the SM proceeds by the Higgs mechanism of spontaneous symmetry breaking [22]. This mechanism requires the introduction of a scalar field which transforms as a 


\begin{tabular}{|c||c|c|c|c|c|}
\hline \multicolumn{1}{|c||}{} & \multicolumn{5}{c|}{ Standard Model } \\
\hline fermion & $Q_{L}^{m}$ & $u_{R}^{m}$ & $d_{R}^{m}$ & $L_{L}^{m}$ & $e_{R}^{m}$ \\
\hline$\left(S U(3)_{c} \times S U(2)_{L}\right)_{Y}$ & $(\mathbf{3}, \mathbf{2})_{\frac{1}{3}}$ & $(\mathbf{3}, \mathbf{1})_{\frac{4}{3}}$ & $(\mathbf{3}, \mathbf{1})_{\frac{-2}{3}}$ & $(\mathbf{1}, \mathbf{2})_{-\mathbf{1}}$ & $(\mathbf{1}, \mathbf{1})_{-2}$ \\
\hline
\end{tabular}

Table 1.1: $S U(3)_{c} \otimes S U(2)_{L} \otimes U(1)_{Y}$ representations of the SM fermions. The index $m$ runs over 3 generations of quarks and leptons. Subindices L and R distinguish left-handed and right-handed fields respectively. The subscript Y stands for the weak hypercharge.

doublet under the $S U(2)_{L}$ and acquires a non-vanishing vacuum expectation value (vev). The masses of the gauge bosons are determined by the value of the Higgs field vev, while fermion masses as well as the Higgs boson mass, and the Higgs vev itself remain free parameters.

Results from the SLC, LEP, Tevatron, and other colliders reveal no significant deviations from the SM predictions so far. Theorists, however, regard the SM as an effective theory, which is adequate only at the presently explored energies and must fail at a certain higher energy scale $\Lambda$. At the very least, the SM, which does not incorporate gravity, must become inadequate at the Planck scale $\sim 10^{19} \mathrm{GeV}$ where gravity becomes comparable in strength to other forces. Moreover, there is a strong theoretical bias to believe that the SM breaks down much earlier, at the $\mathrm{TeV}$ scale. The argument is based on the inability of the SM to adequately explain the nature of the electroweak symmetry breaking (EWSB) that generates masses for the SM particles and also on the so called hierarchy problem that arises when one considers radiative corrections to the mass of the Higgs particle. Much of the theoretical data favors $\Lambda \sim 10 \mathrm{TeV}$.

On the experimental side, there is strong evidence for oscillations of atmo- 
spheric neutrinos [24] (originating from electromagnetic cascades initiated by cosmic rays in the upper atmosphere) and solar neutrinos [25]. The oscillation mechanism can be explained by the introduction of a non-vanishing mass for a neutrino particle, in contrast to the zero mass neutrinos of the SM. Also astrophysical observations unambiguously show that ordinary matter constitutes only about $5 \%$ of the energy density of the universe, while the remaining $95 \%$ is distributed among dark matter $(22 \%)$ and dark energy (73\%). Most of the available results favor dark matter to be a stable heavy (i.e. with the mass of order $100 \mathrm{GeV}$ ) particle that interacts weakly with the SM particles. Such a particle is commonly called a weakly interacting massive particle (WIMP). The concepts of dark energy and dark matter do not find an explanation in the framework of the SM of particle physics and therefore provide a great incentive for explorations of various extensions of the Standard Model. In the next section we discuss astrophysical evidence that convinces us of the existence of dark matter particles and we outline the major properties of those particles as suggested by the astrophysical data.

\subsection{Dark Matter in the Universe}

It is remarkable that we still don't understand the nature of the primary source of matter in our Universe. Most of the matter that forms galaxies, galaxy clusters and superclusters is dark - it does not emit or absorb radiation at any wavelength. Yet its gravitational pull determines the development of structure on all the observed cosmic scales from tiny dwarf galaxies to the large spiral galaxies like our Milky Way and on to the largest structures observed. Over the last two decades the evidence for dark matter has become incontrovertible. The question has changed from "Does 


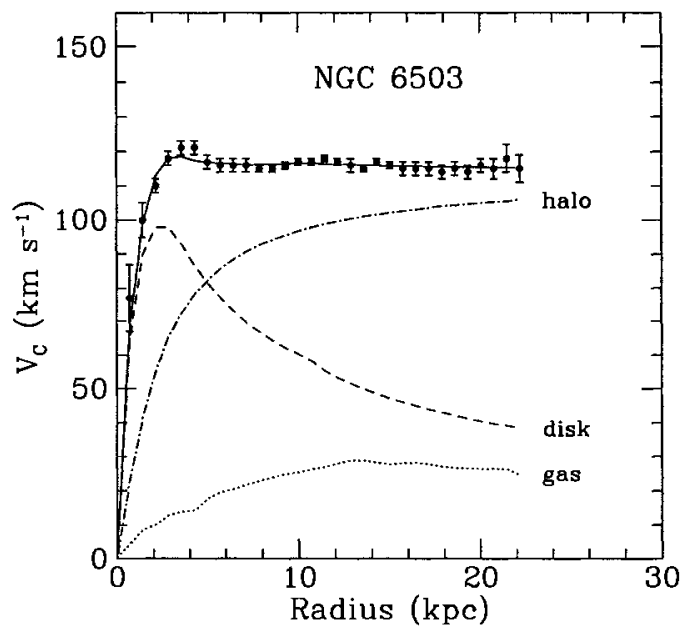

Figure 1.1: Rotation curve of the spiral galaxy NGC 6503. The dotted, dashed and dash-dotted lines are the contributions of gas, disk and dark matter, respectively. Figure adopted from Ref. [26].

dark matter exist?" to "What is dark matter?".

\subsubsection{Astrophysical evidence for dark matter}

The most convincing and direct evidence for dark matter comes from the observations of the rotation curves of galaxies - the dependence of the circular velocities of stars and gas in a galaxy as a function of their distance from the galactic center [26]. Remarkably, observed rotation curves usually exhibit a characteristic flat behavior at the edge of the visible disks and beyond (see Fig. 1.1), whereas in Newtonian dynamics the circular velocity is expected to be falling as $v(r) \propto 1 / \sqrt{r}$ beyond the edge of luminous matter. The fact that $v(r)$ is approximately constant implies the existence of a dark matter halo with $M(r) \propto r$ and $\rho(r) \propto 1 / r^{2}$.

Another important piece of evidence for dark matter comes from gravitational 
lensing effects. According to General Relativity, the path of light can be bent by the presence of a large mass source. Lensing effects are observed when the light on its way to a telescope from a distant galaxy or galaxy cluster passes near a large distribution of matter. The lensing effect allows an estimate of the amount of matter that lies between the observer and the light source. Numerous studies of this gravitational lensing strongly confirm the presence of dark matter and also allow us to map the spatial distribution of dark matter [27].

Further evidence comes from the distribution of hot intergalactic gas inside galaxy clusters. Results from the Chandra X-ray and XMM-Newton space observatories [28] provided a map of the distribution of hot, x-ray emitting gas bound to clusters. The visible mass in the observed clusters can not provide enough gravitational pull to bind the observed gas, while the introduction of dark matter into the analysis allows an explanation of all the observed data. A recent example of gas dynamics inside galaxy clusters comes from the observations of the so-called bullet cluster (1E 0657-56) [29]. The combination of the x-ray observations of the hot gas and the lensing observation of the matter distribution reveal an actual separation of dark matter from the gaseous halo made of baryonic matter. This separation is expected if a vast halo of non-interacting dark matter accompanies the luminous matter and it finds an excellent agreement with the results of recent simulations based on the CDM paradigm [30].

Convincing evidence for dark matter also comes from the theoretical side. First, if the mass density contributed by the visible matter were the major component of the mass density in the universe, the duration of the structure formation epoch would be too short to account for the observed distributions of galaxies and clusters and also would considerably alter the fluctuations of the cosmic microwave 
background (CMB) radiation. The peaks and troughs in the CMB power spectrum are very sensitive to the composition of the universe and provide an independent confirmation of the $\Lambda$ CDM model $[2,31]$. Observations of large scale structure match extremely well with the results of cosmological simulations of the universe including both WIMP dark matter and dark energy [32]. Furthermore, dark matter is essentially required by the inflationary paradigm, which provides the most compelling explanation of a variety of astrophysical phenomena. Inflation predicts the universe to be flat, that is, the energy density of the universe to be equal to the critical density. However, stars, dust and interstellar gas clouds can not provide enough matter to account for the flatness of universe; in fact these sources constitute only about $5 \%$ of the required energy density.

Combined astrophysical data favors dark matter consisting of massive, electrically and color neutral, weakly interacting particles that were produced as thermal relics with non-relativistic velocities in the very early universe [33]. Properties of dark matter particles obtained from the astrophysical observations can provide important guidance for extensions of the SM of particle physics. One of the most important and stringent constraints on any model beyond the SM comes from matching the observed abundance of dark matter in the universe to the relic abundance calculated within the model. In the next section we describe the physics of the relic density calculations.

\subsubsection{Relic abundance of dark matter}

The amount of dark matter today, the relic density, has been measured with good precision by WMAP as $0.094<\Omega_{D M} h^{2}<0.126$ [2]. Here $\Omega_{D M}$ is the ratio of dark matter density to the critical density of the universe and $h$ parametrizes the Hubble 
constant today. From a theoretical side we can calculate the relic density of dark matter in terms of the major parameters of any models that produce a stable WIMP and hence impose stringent constraints on the allowed parameter space of the models.

In the early universe, dark matter particles were present in large numbers in thermal equilibrium with the rest of the cosmic plasma; as the universe cooled, they could reduce their density only through pair annihilation. As their density decreases, however, it becomes more and more difficult for particles to find partners to annihilate with, and the comoving density becomes constant, known as "freeze-out". Therefore, the present day density of dark matter particles is completely determined at the time of freeze-out.

The dynamics of various particle species in the early universe is conveniently described by the distribution function $f(\mathbf{p}, \mathbf{x})$, that counts the number of particles in an infinitesimal region in phase space around position $\mathbf{x}$ and momentum $\mathbf{p}$ or, alternatively, by the comoving number density $n$ which is just an integral of the distribution function over the available phase space [34]. The evolution of the phase space distribution function, $f(\mathbf{p}, \mathbf{x})$, is described by the Boltzmann equation

$$
\mathbf{L}[f]=\mathbf{C}[f],
$$

where $\mathbf{L}$ is the Liouville operator, and $\mathbf{C}$ is the collision operator that contains all possible collision terms. These terms, in general, are complicated functionals of the distribution functions of the various constituents of the universe. However, in most cases of interest, the collision term can be reduced to the interaction between a small number of particle species and hence can be written in a relatively simple form.

In the generic WIMP scenario, two heavy dark matter particles $\chi$ annihilate producing two light, essentially massless, particles $l$ that are tightly coupled to the rest 
of the cosmic plasma and therefore are maintained in complete equilibrium. There remains then only one unknown in the Boltzmann equation $-n_{\chi}$, the abundance of the WIMP. After some manipulation, the Boltzmann equation can be written as an equation for $n_{\chi}$

$$
\frac{d n_{\chi}}{d t}+3 H n_{\chi}=-\langle\sigma v\rangle\left(n_{\chi}^{2}-\left(n_{\chi}^{e q}\right)^{2}\right)
$$

where $\langle\sigma v\rangle$ is the total thermally averaged annihilation cross section multiplied by the relative velocity, $H$ is the Hubble constant, and $n_{\chi}^{e q}$ is the number density of dark matter particles at thermal equilibrium. For massive particles, namely in the non-relativistic limit, and in the Maxwell-Boltzmann approximation, one has

$$
n_{\chi}^{e q}=g\left(\frac{m T}{2 \pi}\right)^{3 / 2} e^{-m / T}
$$

where $m$ is the particle mass and $T$ is the temperature. We next introduce the variables

$$
Y_{\chi} \equiv \frac{n_{\chi}}{s} \quad, \quad Y_{\chi}^{e q} \equiv \frac{n_{\chi}^{e q}}{s}
$$

where $s$ is the entropy density $s=2 \pi^{2} g_{*} T^{3} / 45$ and $g_{*}$ counts the number of relativistic degrees of freedom and is approximately equal to the number of bosonic relativistic degrees of freedom plus $7 / 8$ of the fermionic relativistic degrees of freedom (a plot of $g_{*}$ as a function of time can be found for instance in [35]). Using the conservation of entropy per co-moving volume $\left(s a^{3}=\right.$ constant $)$, it follows that $\dot{n}_{\chi}+3 H n_{\chi}=s \dot{Y}_{\chi}$ and Eq. 1.3 reads

$$
s \dot{Y}_{\chi}=-\langle\sigma v\rangle s^{2}\left(Y_{\chi}^{2}-\left(Y_{\chi}^{e q}\right)^{2}\right)
$$

We can further introduce the dimensionless variable $x \equiv \frac{m}{T}$, and rewrite Eq. 1.6 as

$$
\frac{d Y_{\chi}}{d x}=-\frac{\langle\sigma v\rangle s}{H x}\left(Y_{\chi}^{2}-\left(Y_{\chi}^{e q}\right)^{2}\right)
$$

It is conventional, to approximate $\langle\sigma v\rangle$ with a truncated Taylor expansion in powers 
of $v^{2}$ or equivalently in inverse powers of $x$.

$$
\langle\sigma v\rangle \approx a+b \frac{1}{x}
$$

The coefficients $a$ and $b$ in this expansion depend on the actual physics of a given extension of the SM and can be generically written in terms of the invariant amplitudes of a particular annihilation process [36,37]. Using this expansion our final version of Eq. 1.7 in terms of the variable $\Delta_{\chi}=Y_{\chi}-Y_{\chi}^{e q}[38]$ is:

$$
\Delta_{\chi}^{\prime}=-Y_{\chi}^{e q \prime}-f(x) \Delta_{\chi}\left(2 Y_{\chi}^{e q}+\Delta_{\chi}\right)
$$

where the prime denotes $d / d x$ and where

$$
f(x)=\sqrt{\frac{\pi g_{*}}{45}} m M_{P l}(a+b / x) x^{-2} .
$$

Following [33] we can introduce the quantity $x_{F} \equiv m / T_{F}$, where $T_{F}$ is the freeze-out temperature of the relic particle, and we notice that Eq. 1.9 can be solved analytically in the two extreme regions $x \ll x_{F}$ and $x \gg x_{F}$ as

$$
\begin{array}{llll}
\Delta_{\chi}=-\frac{Y_{\chi}^{e q \prime}}{2 f(x) Y_{\chi}^{e q}} & \text { for } & x \ll x_{F} \\
\Delta_{\chi}^{\prime}=-f(x) \Delta_{\chi}^{2} & \text { for } & x \gg x_{F} .
\end{array}
$$

These regions correspond to times long before freeze-out and long after freeze-out, respectively. Integrating the last equation between $x_{F}$ and $\infty$ and using $\Delta_{\chi}\left(x_{F}\right) \gg$ $\Delta_{\chi}(\infty)$, we can derive the value of $\Delta_{\chi}(\infty)$ and arrive at

$$
\left[Y_{\chi}(\infty)\right]^{-1}=\sqrt{\frac{\pi g_{*}}{45}} M_{P l} m x_{F}^{-1}\left(a+b / x_{F}\right) .
$$

The present day density of a generic relic dark matter particle, $\chi$, is simply given by $\rho_{\chi}=m_{\chi} n_{\chi}=m_{\chi} s_{0} Y_{\chi}(\infty)$, where $s_{0}=2889.2 \mathrm{~cm}^{-3}$ is the present entropy density [39]. The relic density can finally be expressed in terms of the critical density

$$
\Omega_{\chi} h^{2} \approx \frac{1.07 \times 10^{9} \mathrm{GeV}^{-1}}{M_{P l}} \frac{x_{F}}{\sqrt{g_{*}}} \frac{1}{\langle\sigma v\rangle},
$$


where $g_{*}$ and $\langle\sigma v\rangle$ are evaluated at the freeze-out temperature. It is conventional to write the relic density in terms of the Hubble parameter, $h=H_{0} / 100 \mathrm{~km} \mathrm{~s}^{-1} \mathrm{Mpc}^{-1}$.

The freeze-out temperature $x_{F}$ can be estimated through iterative solution of the equation [33]

$$
x_{F}=\ln \left[c(c+2) \sqrt{\frac{45}{8}} \frac{g}{2 \pi^{3}} \frac{m M_{P l}\langle\sigma v\rangle}{g_{*}^{1 / 2} x_{F}^{1 / 2}}\right],
$$

where $c$ is a constant of order one determined by matching the late-time and earlytime solutions.

It is important to note that the approximation introduced in Eq. 1.8 is not always justified [40]. Dramatic changes to dark matter relic density occur when another particle lies near in mass to the relic particle and shares a quantum number with it. The presence of such a particle can induce resonant enhancements or socalled coannihilations to $\langle\sigma v\rangle$, thus making the simple Taylor expansion invalid. The approximation of Eq. 1.8 also fails when the annihilation takes place close to the pole of the cross section, in which case a special numerical treatment is required.

Quite remarkably, the relic density of dark matter $\Omega_{\chi}$ does not explicitly depend on the mass of the WIMP. It is mainly the model dependent annihilation cross section which determines the relic abundance. In the next Chapter we discuss dark matter candidates that arise in the Simplest Little Higgs with T-parity and Littlest Higgs with T-parity extensions of the SM and we specify annihilation processes that determine the relic abundance in each model. 


\section{Chapter 2}

\section{Dark matter in Little Higgs models}

Little Higgs models [6-11] offer an interesting alternative to supersymmetry in solving various problems of the SM. For instance, the hierarchy problem is resolved by introducing new heavy particles at the $\mathrm{TeV}$ scale that cancel quadratic divergences of the Higgs boson mass that arise in the SM from one-loop radiative corrections. This cancellation occurs between particles with the same statistics in Little Higgs models, as opposed to the case of supersymmetry where divergences are canceled by opposite statistics partners of the SM particles. The original little Higgs models [9,41,42], however, suffered from stringent constraints from the electroweak precision tests (EWPT) which required the introduction of fine tuning. An elegant solution to this problem is to introduce a discrete symmetry (like the R-parity in supersymmetry) called Tparity [15]. T-parity not only remedies the problem of precision electroweak constraints, but also leads to the appearance of promising dark matter candidates. We must note, that recently it has been pointed out that T-parity in general can be violated by anomalies [43], making the lightest T-odd particle unstable. However, such violation strongly depends on the UV completion of the Little Higgs and may 
not be realized in nature [44]. Consequently, we consider models respecting T-parity here. In this Chapter, we describe two Little Higgs models incorporating T-parity: the Littlest Higgs with T-parity model is discussed in Section 2.1, while the Simplest Little Higgs with T-parity model is presented in Section 2.2.

\subsection{Littlest Higgs with T-parity}

The Littlest Higgs with T-parity model (LHT) [13-16] is a modification of the original Littlest Higgs Model [9], which introduces an interesting dark matter candidate as well as remedying several other problems of the Littlest Higgs Model. The original model is based on a non-linear $\sigma$-model describing a global $S U(5) / S O(5)$ symmetry breaking, which occurs at an energy scale $\Lambda \sim 4 \pi f \sim 10 \mathrm{TeV}$. The vev associated with the spontaneous symmetry breaking is parametrized by the $5 \times 5$ matrix $\Sigma_{0}$ of the form [45]

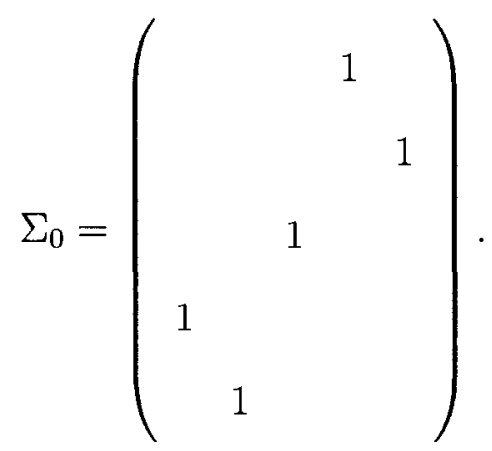

This symmetry breaking also breaks the assumed embedded local gauge symmetry $[S U(2) \otimes U(1)]^{2}$ into its diagonal subgroup $\left[S U(2)_{L} \otimes U(1)_{Y}\right]$, which is just an ordinary standard model gauge group. The scalar fields of the model are parametrized as

$$
\Sigma=e^{i \Pi / f} \Sigma_{0} e^{i \Pi^{T} / f}
$$


where $\Pi$ is the Goldstone boson matrix, built of the 14 broken $S U(5)$ generators $X^{a}$ :

$$
\Pi=\pi^{a} X^{a}=\left(\begin{array}{ccc} 
& h^{\dagger} / \sqrt{2} & \phi^{\dagger} \\
h / \sqrt{2} & & h^{*} / \sqrt{2} \\
\phi & h^{T} / \sqrt{2} &
\end{array}\right) .
$$

The diagonal Goldstone bosons (not shown) are "eaten" to become the longitudinal modes of the heavy partners of the standard model gauge bosons at the scale $f$, as we go to the unitary gauge. The remaining scalar fields are an $S U(2)_{L}$ doublet $h^{T}=\left(h^{+}, h^{0}\right)$, identified as a SM Higgs field, and a triplet $\phi$ that receives a mass at the TeV scale. The gauge-invariant kinetic term of the non-linear $\sigma$-model field $\Sigma$, responsible for the masses of the gauge bosons, can be written as follows

$$
\mathcal{L}_{k i n}=\frac{f^{2}}{8} \operatorname{Tr}\left(D_{\mu} \Sigma\right)\left(D^{\mu} \Sigma\right)^{\dagger}
$$

where the covariant derivative is

$$
D_{\mu} \Sigma=\partial_{\mu} \Sigma-i \sum_{j}\left[g_{j} W_{j}^{a}\left(Q_{j}^{a} \Sigma+\Sigma Q_{j}^{a T}\right)+g_{j}^{\prime} B_{j}\left(Y_{j} \Sigma+\Sigma Y_{j}\right)\right]
$$

with $j=1,2$ and $a=1,2,3 . S U(2)_{j}$ gauge fields in the covariant derivative are $W_{j}^{a}$ and $U(1)_{j}$ gauge fields are $B_{j}$, with the two copies of $S U(2) \otimes U(1)$ embedded into $\mathrm{SU}(5)$ via the generators:

$$
\begin{aligned}
Q_{1}^{a} & =\left(\begin{array}{ccc}
\sigma^{a} / 2 & 0 & 0 \\
0 & 0 & 0 \\
0 & 0 & 0
\end{array}\right), \quad Y_{1}=\operatorname{diag}(3,3,-2,-2,-2) / 10 \\
Q_{2}^{a} & =\left(\begin{array}{ccc}
0 & 0 & 0 \\
0 & 0 & 0 \\
0 & 0 & -\sigma^{a *} / 2
\end{array}\right), \quad Y_{2}=\operatorname{diag}(2,2,2,-3,-3) / 10
\end{aligned}
$$


where $\sigma^{a}$ are the Pauli matrices. This kinetic term leads to the heavy masses of the additional gauge bosons $A_{h}, Z_{h}$ and $W_{h}^{ \pm}$:

$$
M_{A_{h}}=\frac{g^{\prime} f}{\sqrt{5}}, \quad M_{Z_{h}} \approx M_{W_{h}}=g f,
$$

where

$$
g=\frac{g_{1} g_{2}}{\sqrt{g_{1}^{2}+g_{2}^{2}}}, \quad g^{\prime}=\frac{g_{1}^{\prime} g_{2}^{\prime}}{\sqrt{g_{1}^{\prime 2}+g_{2}^{\prime 2}}} .
$$

After electroweak symmetry breaking, masses of the new heavy gauge bosons as well as the SM gauge boson masses receive $v^{2} / f^{2}$ corrections, however, these corrections have a negligible impact on dark matter phenomenology and therefore are not considered in this study.

T-parity is naturally introduced in this framework [15], by requiring SM particles to be even and the particles of a new sector to be T-parity odd. The action of T-parity is defined as $A_{1} \rightarrow A_{2}$, where $A_{1}, A_{2}$ are the gauge fields corresponding to $[S U(2) \otimes U(1)]_{1}$ and $[S U(2) \otimes U(1)]_{2}$ symmetries, respectively. The Lagrangian Eq. 2.4 is invariant under T-parity if $g_{1}=g_{2}=\sqrt{2} g$ and $g_{1}^{\prime}=g_{2}^{\prime}=\sqrt{2} g^{\prime}$. In fact we can identify $g$ and $g^{\prime}$ with the SM $S U(2)_{L}$ and $U(1)_{Y}$ couplings. In order to incorporate T-parity systematically one needs to add new fermion fields [16] that obtain masses $M_{f} \sim \sqrt{2} k_{f} f$, with $k_{f}$ - the Yukawa type couplings introduced in the interaction of new fermions with the scalar fields. In this thesis we chose to work with the universal $k_{f}=1$ value of the Yukawa coupling to the new fermions. This choice leads to decoupling of the new heavy fermions (except for the new heavy top quarks, whose masses are determined from the cancellation of the SM top quark quadratic divergence), and allows us to concentrate on the phenomenology of the gauge and heavy fermion sectors that are common to all implementations of the Littlest Higgs with T-parity. Analysis of the LHT collider phenomenology for the case of varying values of $k_{l}$ and $k_{q}$ can be found in [46]. 
Typically, the lightest T-odd particle in the Littlest Higgs with T-parity model is a heavy partner of the photon $A_{h}$, which has mass $M_{A_{h}}=f g^{\prime} / \sqrt{5} \approx 0.11 f$. The only direct coupling of the heavy photon to the SM particles is via Higgs exchange, resulting in weak-strength cross sections for $A_{h}$ annihilation into SM states. The heavy photon then provides a great candidate for the WIMP and therefore has received a lot of attention in the literature [16,47-49]. The Higgs boson mediated coupling of the heavy photon to the SM particles leads to a relatively small set of dark matter annihilation channels that determine the relic density and, thus, allow us to constrain the model. Fig. 2.1 shows Feynman diagrams of the major processes that contribute to the dark matter annihilation in the early universe.

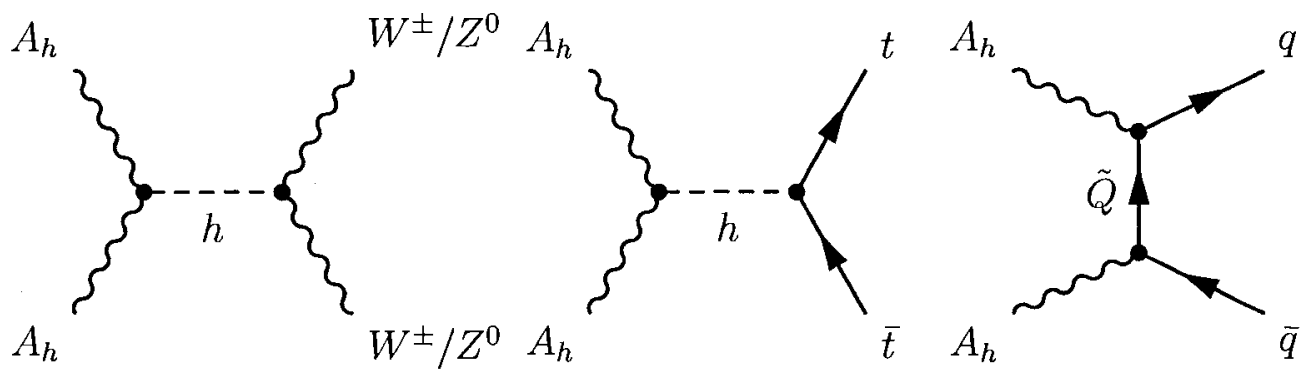

Figure 2.1: The leading annihilation processes which maintain the heavy photon of the Littlest Higgs with T-parity model in equilibrium with the rest of the cosmic plasma in the early universe.

In the middle diagram we show only the annihilation channel into top quarks since annihilation into the light quarks and leptons is strongly suppressed by Yukawa coupling. In the rightmost diagram the annihilation goes through the exchange of a heavy partner of the SM quark. This process has a non-negligible contribution only when masses of the heavy quarks are small enough, i.e. $M_{\tilde{Q}} \propto M_{A_{h}}$, in which case one also needs to consider various coannihilation processes. The relic density constraint for this case can be found in the journal version of [16] and the collider 
phenomenology for the heavy quarks is discussed in [48]. We also note that if the Higgs boson is lighter than the dark matter particle there will be a contribution from $A_{h} A_{h} \rightarrow h h$ annihilation.

For our choice of the heavy fermion masses, only the s-channel Higgs exchange contributes to dark matter annihilation and the cross section exhibits a resonant behavior when the mass of $A_{h}$ approaches half the mass of the Higgs boson. This resonance enhancement allows the right value of the relic density on both sides of the Breit-Wigner peak. Relic density constraints for this case can be found in the preprint version of [16] and can be conveniently parametrized by [47]

$$
m_{h} \approx 24+2.38 M_{A_{h}} \text { and } m_{h} \approx-83+1.89 M_{A_{h}} .
$$

These stringent constraints from the relic density calculations allow one to make specific predictions for the collider signatures of the Littlest Higgs with Tparity model. In chapter 3 we will outline major channels of dark matter production at the LHC.

\subsection{Simplest Little Higgs with T-parity}

The Simplest Little Higgs with T-parity model [12] is an alternative realization of the Little Higgs mechanism [6-11] that has received less attention in the literature so far. It is constructed by enlarging the SM $S U(2)_{L} \otimes U(1)_{Y}$ gauge group to $S U(3)_{w} \otimes$ $U(1)_{X}$ in a minimal way. This entails enlarging the SU(2) doublets of the SM to SU(3) triplets, adding the additional SU(3) gauge bosons, and writing SU(3) invariant interactions in a way that reproduces all the SM couplings when restricted to SM fields. The $S U(3)_{W} \otimes U(1)_{X}$ gauge symmetry is broken down to the SM electroweak 
gauge group by two complex scalar fields $\Phi_{1,2}$, which are triplets under the $\mathrm{SU}(3)$ with aligned vevs $f_{1,2}$. When the scalar fields acquire vevs, the initial global symmetry $\left[S U(3)_{W} \otimes U(1)_{X}\right]^{2}$ is spontaneously broken to $\left[S U(2) \otimes U(1)_{X}\right]^{2}$. At the same time, the global symmetry is explicitly broken to its diagonal subgroup $S U(3) \otimes U(1)$ by the gauge interactions.

The scalar fields are conveniently parameterized by a nonlinear sigma model of the $\left[S U(3) \otimes U(1)_{X}\right]^{2} /\left[S U(2) \otimes U(1)_{X}\right]^{2}$ symmetry breaking as

$$
\Phi_{1}=e^{i \ominus \frac{f_{2}}{f_{1}}}\left(\begin{array}{c}
0 \\
0 \\
f_{1}
\end{array}\right), \quad \Phi_{2}=e^{-i \Theta \frac{f_{1}}{f_{2}}}\left(\begin{array}{c}
0 \\
0 \\
f_{2}
\end{array}\right)
$$

where

$$
\Theta=\frac{1}{f}\left[\left(\begin{array}{ccc}
\frac{\eta}{\sqrt{2}} & 0 & 0 \\
0 & \frac{\eta}{\sqrt{2}} & 0 \\
0 & 0 & \frac{\eta}{\sqrt{2}}
\end{array}\right)+\left(\begin{array}{ccc}
0 & 0 & \\
0 & 0 & \\
h^{\dagger} & 0
\end{array}\right)\right] \text { and } f^{2}=f_{1}^{2}+f_{2}^{2} .
$$

Here $\eta$ is a real scalar field and $h=\left(h^{0}, h^{-}\right)^{T}$ is an $S U(2)$ doublet which can be identified as the SM Higgs. The remaining 5 degrees of freedom of the symmetry breaking are "eaten" by the additional heavy gauge bosons and therefore are omitted from 2.10 .

Scalar field interactions with the gauge boson sector and the masses of gauge bosons are determined by the standard kinetic term for the $\Phi_{i}[50]$ :

$$
\mathcal{L}_{\Phi}=\left|\left(\partial_{\mu}+i g A_{\mu}^{a} T^{a}-\frac{i g_{x}}{3} B_{\mu}^{x}\right) \Phi_{i}\right|^{2}
$$

where the $\mathrm{SU}(3)$ gauge coupling $g$ is equal to the $\mathrm{SM} \mathrm{SU}(2)_{L}$ gauge coupling and the $\mathrm{U}(1)_{X}$ gauge coupling $g_{x}$ is fixed in terms of $g$ and the weak mixing angle $t_{W} \equiv \tan \theta_{W}$ 
by

$$
g_{x}=\frac{g t_{W}}{\sqrt{1-t_{W}^{2} / 3}} .
$$

The gauge bosons corresponding to the broken generators get masses of order $f \sim$ $\mathrm{TeV}$ and consist of a $Z_{h}$ boson, which is a linear combination of $A^{8}$ and $B^{x}$

$$
Z_{h}=\frac{1}{\sqrt{3}}\left(\sqrt{3-t_{W}^{2}} A^{8}+t_{W} B^{x}\right),
$$

and a complex $\mathrm{SU}(2)_{L}$ doublet $\left(Y^{0}, X^{-}\right)$

$$
X^{-}=\frac{A^{6}-i A^{7}}{\sqrt{2}}, \quad Y^{0}=\frac{A^{4}-i A^{5}}{\sqrt{2}} .
$$

The SM mass eigenstates $W^{ \pm}, Z^{0}$, and $A$ are also expressed in terms of the gauge fields:

$$
\begin{gathered}
W^{ \pm}=\frac{A^{1} \mp i A^{2}}{\sqrt{2}}, \\
Z^{0}=A^{3} c_{W}+\frac{t_{W}}{\sqrt{3}}\left(A^{8} s_{W}-B^{x} c_{W} \sqrt{3-t_{W}^{2}}\right) \\
A=A^{3} s_{W}-A^{8} \frac{s_{W}}{\sqrt{3}}+B^{x} \frac{c_{W}}{\sqrt{3}} \sqrt{3-t_{W}^{2}} .
\end{gathered}
$$

As in the SM, masses of the heavy gauge bosons are determined by the symmetry breaking vev and can be written as:

$$
M_{Z_{h}}=\frac{\sqrt{2} g f}{\sqrt{3-t_{W}^{2}}}, \quad M_{X^{ \pm}}=M_{Y^{0}}=\frac{g f}{\sqrt{2}}
$$

The spontaneous electroweak symmetry breaking $S U(2) \times U(1) / U(1)_{\text {em }}$ by the vev of the SM Higgs introduces corrections to the masses of the gauge bosons. For example, the charged heavy bosons $X^{ \pm}$become slightly lighter than the neutral gauge boson $Y^{0}$. However, for our studies of dark matter physics, we are interested only in the first order effects and therefore will disregard $(v / f)^{2}$ corrections to masses and to the couplings in the Feynman rules. 
There are two possible gauge charge assignments for fermions in the Simplest Little Higgs model. The first (universal embedding) has heavy partners of the top, charm and up quarks, with all generations carrying identical quantum numbers. However, this embedding is known to be anomalous and also appears to be ruled out by precision electroweak constraints [42]. In the second scenario (anomaly-free embedding) the top, strange and down quarks have heavy partners. The first two generations of quarks transform under the antifundamental $\overline{3}$ representation of the $\mathrm{SU}(3)$, while the third generation quarks and leptons transform as 3's [50]:

$$
\begin{array}{cc}
L_{m}^{T}=(\nu, e, i N)_{m}, & i e_{m}^{c}, i N_{m}^{c}, \\
Q_{1}^{T}=(d,-u, i D), & i d^{c}, i u^{c}, i D^{c}, \\
Q_{2}^{T}=(s,-c, i S), & i s^{c}, i c^{c}, i S^{c} \\
Q_{3}^{T}=(t, b, i T), & i t^{c}, i b^{c}, i T^{c},
\end{array}
$$

where $\mathrm{m}$ is the generation index, and $u^{c}, d^{c}, \ldots$ are the right-handed Weyl fermions invariant under $S U(3)_{W}$ that marry corresponding components of the $S U(3)_{W}$ triplets to produce fermion masses. Hypercharge assignments and representations of the fundamental fields in the anomaly-free embedding are summarized in Table 2.1. We will consider only this case.

As in the Littlest Higgs model we can introduce a discrete symmetry ( $T$ parity) into the model to avoid precision electroweak constraints and to produce a heavy stable particle that will become a dark matter candidate [12]. A straightforward way to implement T-parity into the Simplest Little Higgs model is to start with the fermionic sector. The action of the T-parity operator on the fermion triplets can be defined as [12]

$$
\Psi_{Q, L} \rightarrow-\hat{\Omega} \Psi_{Q, L}
$$




\begin{tabular}{|c||c|}
\hline & Anomaly-free embedding \\
\hline fermion & $\left(S U(3)_{c} \times S U(3)_{w}\right)_{U(1)_{X}}$ \\
\hline$Q_{1,2}$ & $(\mathbf{3}, \overline{\mathbf{3}})_{0}$ \\
\hline$Q_{3}$ & $(\mathbf{3}, \mathbf{3})_{\frac{1}{3}}$ \\
\hline$u_{a}^{c}, T^{c}$ & $(\overline{\mathbf{3}}, \mathbf{1})_{\frac{-2}{3}}$ \\
\hline$d_{a}^{c}, D^{c}, S^{c}$ & $(\overline{\mathbf{3}}, \mathbf{1})_{\frac{1}{3}}$ \\
\hline$L_{m}$ & $(\mathbf{1}, \mathbf{3})_{\frac{-1}{3}}$ \\
\hline$N_{m}^{c}$ & $(\mathbf{1}, \mathbf{1})_{0}$ \\
\hline$e_{m}^{c}$ & $(\mathbf{1}, \mathbf{1})_{\mathbf{1}}$ \\
\hline
\end{tabular}

Table 2.1: The $S U(3)_{c} \otimes S U(3)_{W} \otimes U(1)_{X}$ representations of the fermions in the anomaly-free embedding of the Simplest Little Higgs with T-parity model. Index $a$ runs over the first two generations of quarks, while $m$ runs over all three generations of leptons.

where $\hat{\Omega}=\operatorname{diag}(-1,-1,1)$. For the scalar fields the transformation is

$$
\Phi_{1} \rightarrow \hat{\Omega} \Phi_{2}
$$

which immediately requires $f_{1}=f_{2}=f / \sqrt{2}$. This definition of T-parity also determines the following transformation rules for the gauge boson sector, which follow from the requirement of the T-parity invariance for the kinetic term 2.11.

$$
A_{\mu} \rightarrow \hat{\Omega} A_{\mu} \hat{\Omega}
$$

It is important to note that, under this transformation, the new neutral gauge boson $Z_{h}$ remains even, and hence its mass is required to be relatively heavy in order 
to avoid the precision electroweak constraints.

T-parity invariance requires that both scalar triplets $\Phi_{1}$ and $\Phi_{2}$ couple to the fermion fields with equal strength. This introduces major modifications to the standard Simplest Little Higgs model. Yukawa interactions in the leptonic sector can be written as

$$
\mathcal{L}_{l}=i \lambda_{N_{m}} N_{m}^{c}\left(\Phi_{1}^{\dagger}+\Phi_{2}^{\dagger}\right) L_{m}+\frac{i \lambda_{e}^{m}}{\Lambda} e_{m}^{c} \epsilon_{i j k} \Phi_{1}^{i} \Phi_{2}^{j} L_{m}^{k}+\text { h.c. }
$$

where $m=1,2,3$ is a generation index and $i, j, k=1,2,3$ are $\mathrm{SU}(3)$ indices. Yukawa Lagrangians for the third generation of quarks and for the first two generations are, respectively,

$$
\begin{gathered}
\mathcal{L}_{3}=i \lambda_{t}\left(t_{L}^{c}\left(\Phi_{2}^{+}-\Phi_{1}^{+}\right)+t_{H}^{c}\left(\Phi_{2}^{+}+\Phi_{1}^{+}\right)\right) Q_{3}+\frac{\lambda_{b}}{\Lambda} i b^{c} \epsilon_{i j k} \Phi_{1}^{i} \Phi_{2}^{j} Q_{3}^{k}+\text { h.c. } \\
\mathcal{L}_{1,2}=i \lambda_{D}^{n} Q_{n}^{T}\left(\Phi_{1}+\Phi_{2}\right) d_{H}^{n c}+i \lambda_{d}^{n} Q_{n}^{T}\left(\Phi_{1}-\Phi_{2}\right) d_{L}^{n c}+\frac{\lambda_{u}^{n}}{\Lambda} i u_{n}^{c} \epsilon_{i j k} \Phi_{1}^{* i} \Phi_{2}^{* j} Q_{n}^{k}+\text { h.c. }
\end{gathered}
$$

where $n=1,2 ; i, j, k=1,2,3$ are $\mathrm{SU}(3)$ indexes; $t_{L}^{c}$ and $t_{H}^{c}$ are T-even and T-odd linear combinations of $t^{c}$ and $T^{c}$, while $d_{H}^{n c}$ and $d_{L}^{n c}$ are linear combinations of $d^{c}$ and $D^{c}$ for $n=1$ and of $s^{c}$ and $S^{c}$ for $n=2$; and $u_{m}^{c}$ runs over all the up-type conjugate quarks $\left(u^{c}, c^{c}, t^{c}\right)$. Also, we are neglecting flavor mixing here.

The requirement of T-invariance leads to a simple relation for the heavy top mass:

$$
M_{T}=M_{t} \frac{\sqrt{2} f}{v}
$$

As in the case of the Littlest Higgs with T-parity the masses of other new fermions come from the Yukawa interaction in the form $M_{f}=\sqrt{2} \lambda_{f} f$. We choose a universal value of the Yukawa coupling $\lambda$ for all new fermions, except for the first generation heavy neutrino. In this work we choose fermion masses as model parameters instead of the Yukawa couplings as a theoretical input of all the calculations. We also note that a more general study without decoupling any of the fermions should be undertaken. 
Introduction of T-parity makes the lightest T-odd particle (LTP) stable. Analysis of the T-odd mass eigenstates shows that, unlike the Littlest Higgs model, the Simplest Little Higgs does not allow a new heavy gauge boson as an LTP, since the lightest new gauge boson $X^{ \pm}$is charged and therefore cannot be a valid dark matter candidate. There are two neutral heavy particles that should be considered: a heavy neutrino $N$ and a new scalar $\eta$. The mass of $\eta$ depends on the mass of the SM Higgs boson as well as on the masses of other heavy particles through the ColemanWeinberg potential [50] and is generally of order $700 \mathrm{GeV}$. Although it is possible to make $\eta$ a LTP it requires significant fine tuning in the parameter space. Moreover, the coupling of $\eta$ to other particles in the primordial plasma is usually $f^{2}$ suppressed which makes it practically impossible to satisfy relic density constraints [12]. On the other hand, the mass of a heavy neutrino remains a free parameter in the model and can be chosen to be significantly lighter than the masses of other particles in the model. A heavy neutrino is weakly coupled to the rest of the particles through $Z_{h}$ and $X^{ \pm}, Y$ exchanges and it decouples at the right time to produce the dark matter density observed today. In light of this, we choose a heavy neutrino as a dark matter candidate of the Simplest Little Higgs with T-parity Model.

Annihilation reactions that kept the heavy neutrino coupled to the primordial plasma before decoupling are shown in Fig. 2.2. It is interesting to note that, unlike the case of the heavy photon in the Littlest Higgs model, the annihilation of the heavy neutrino into $W^{ \pm}$and $Z^{0}$ though the s-channel Higgs exchange is strongly suppressed by the couplings as well as by the fermionic nature of the SLHT dark matter. This can be clearly seen from the Feynman rules of the SLHT model provided in Appendix A.

It is important to emphasize that in this thesis we consider additional quarks 

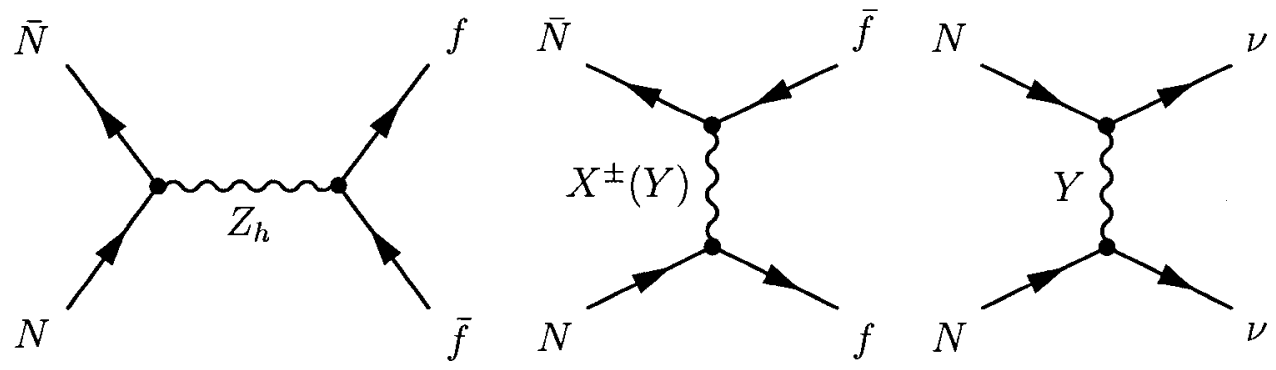

Figure 2.2: Dominant channels for dark matter annihilation in the Simplest Little Higgs with T-parity model. These diagrams provide the largest contributions to the heavy neutrino annihilation cross section for the range of SLHT parameters examined in this thesis.

as well as the second and third generation heavy neutrinos to be much heavier than the LTP and hence effects of coannihilations are ignored. Without coannihilations the dark matter relic density is determined by the $Z_{h}$ s-channel exchange as well as by the t-channel $X^{ \pm}$and $Y$ processes. The t-channel processes are especially important for the heavy LTP. The increase in the cross section caused by the t-channel processes makes the annihilation rate on the right side of the $Z_{h}$ boson peak too large to account for the observed relic density as is depicted in Fig. 2.3. The full set of constraints on the SLHT model that we obtain from the relic density calculations is presented in Fig. 5.1 in Chaper 5 of the thesis. The application of that relic density constraint to dark matter collider phenomenology is discussed in Chapter 4 . 


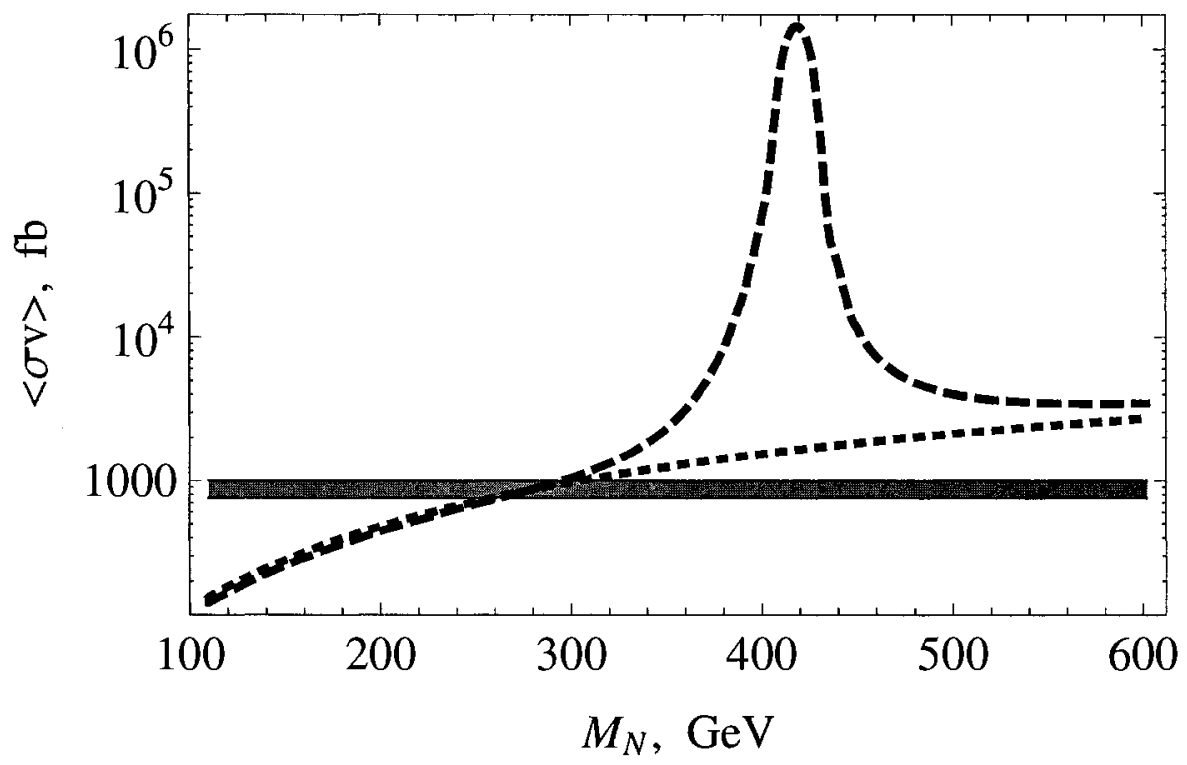

Figure 2.3: t-channel contribution (short-dash line) to the thermally averaged annihilation cross section $\langle\sigma v\rangle$ (dashed line) in the Simplest Little Higgs with T-parity model as a function of dark matter mass. The WMAP allowed region [2] (gray) is also shown for reference. This plot emphasizes the contribution of the t-channel diagrams to the annihilation cross section. 


\section{Chapter 3}

\section{Dark matter properties and the}

\section{formation of complex structures in}

\section{the Universe}

The $\Lambda$ CDM cosmological paradigm uses the idea of perfectly cold, non-interacting dark matter that does not interact with SM particles after freeze-out from the primordial plasma. This idea, however, cannot be correct since all particle dark matter candidates exhibit some interactions. In this section we discuss possibilities to observe the effects of such interactions that may be revealed in various ways at different cosmological scales.

Here we would like to note that, in this Chapter, we present preliminary results of ongoing research; they are meant only to introduce a general idea and illustrate the most salient features of this study. The full version of the results is to be presented elsewhere. 


\subsection{Formalism of structure formation}

Dark matter does not absorb or emit electromagnetic radiation at any wavelength, yet by its gravitational interaction dark matter determines the form of structure on all scales from tiny dwarf galaxies to superclusters. From the very early moments in cosmic history the interplay of dark matter, dark energy and ordinary baryonic matter determines the shape and dynamical properties of all structures we observe today. This interconnection opens an obvious opportunity to study the properties of dark matter particles by analyzing the formation history and the properties of cosmic structures on various scales.

The theory of structure formation $[33,34]$ describes how primordial fluctuations generated by inflation grow into galaxies and clusters of galaxies due to self-gravity. The process of structure formation can be divided into two stages. First, a linear evolution occurs when the amplitudes of the perturbations are small, and the growth of inhomogeneities can be calculated using perturbation theory. With the growth of the perturbations, structure formation proceeds into a non-linear regime that must be analyzed with high-resolution N-body simulations.

The analysis of the linear evolution epoch is based on the Boltzmann equation formalism. In order to trace the evolution of primordial perturbations, one must solve a system of coupled differential equations that generically describes the interactions of all the components of the universe including photons, leptons, baryons, dark matter and dark energy. However, consideration of the interconnection of all types of energy sources in the universe at the same time is a technically insurmountable task. Fortunately, for various periods of cosmic history the impact of different energy sources can be safely neglected. For instance, we can completely ignore the contribution of 
dark energy to the development of structure until redshifts of order 10 and smaller. For the purposes of constraining the dark matter sector, we are also interested only in a number of specific periods of evolution when the contribution of many factors in the collision term of the Boltzmann equation can be neglected.

As we discussed in Section 1.2, the observed number density of dark matter is determined at the very early period when the primordial plasma and dark matter particles are chemically coupled. In the assumption of stable dark matter, the relic density is fixed at the freeze-out (chemical decoupling) that occurs shortly after the temperature of the primordial plasma drops below the mass of dark matter particles $\left(T \propto \frac{1}{10} M_{D M}\right)$. The CDM paradigm assumes that, after freeze-out, dark matter particles are perfectly non-interacting. However, realistic models of particle physics only produce dark matter candidates with some self-interaction and with non-vanishing dark matter - baryon (photon) interaction rates. Because of such interactions dark matter remains kinematically coupled to the primordial photon-lepton plasma long after freeze-out $[20,51]$. This kinetic coupling suppresses the growth of the small scale perturbations and has a profound impact on the formation of the smallest dark matter halos [52].

As in our description of the relic abundance, properties of dark matter particles in the early universe are described by the distribution function $f_{\chi}(\mathbf{x}, \mathbf{q}, \tau)$, where $\mathbf{x}$ are comoving spatial coordinates, $\mathbf{q}=a \mathbf{p}$ are the conjugate momenta, with $a$ being the scale factor of the Universe, and $\tau$ is conformal time. The Boltzmann equation traces the evolution of the distribution function in the perturbed FriedmanRobertson-Walker universe with the line element in the conformal Newtonian gauge written as [34] $d s^{2}=a^{2}\left[-(1+2 \Phi) d \tau^{2}+(1-2 \Psi) d \mathbf{x} \cdot d \mathbf{x}\right]$. After the effects of the 
metric perturbations $\Phi$ and $\Psi$ are included, the Boltzmann equation becomes

$$
\frac{\partial f_{\chi}}{\partial \tau}+\mathbf{v} \cdot \frac{\partial f_{\chi}}{\partial \mathbf{x}}+\left(\dot{\Psi} \mathbf{q}-\epsilon \frac{\partial \Phi}{\partial \mathbf{x}}\right) \cdot \frac{\partial f_{\chi}}{\partial \mathbf{q}}=a C\left[f_{\chi}\right]
$$

where $\mathbf{v}=\mathbf{q} / \epsilon$ is the proper velocity measured by a comoving observer, with $\epsilon \equiv$ $\left(q^{2}+a^{2} m_{\chi}^{2}\right)^{1 / 2}$ the comoving energy [19]. The collision factor $C\left[f_{1 \chi}\right]$ can be written as

$$
\begin{aligned}
C\left[f_{1 \chi}\right]= & \int \frac{d^{3} p_{2}}{E_{2}} \int \frac{d^{3} p_{3}}{E_{3}} \int \frac{d^{3} p_{4}}{E_{4}}\left|\frac{\mathcal{M}}{8 \pi}\right|^{2} \\
& \times \delta^{4}\left(p_{1}+p_{2}-p_{3}-p_{4}\right) \\
& \times\left[f_{3 \chi} f_{4 L}\left(1-\tilde{f}_{2 L}\right)-f_{1 \chi} f_{2 L}\left(1-\tilde{f}_{4 L}\right)\right],
\end{aligned}
$$

where $\mathcal{M}$ is the Lorentz-invariant scattering amplitude, $f_{i \chi} \equiv f_{\chi}\left(\mathbf{p}_{i}\right), E_{i}$ is the energy of particle $i$, and $\tilde{f}_{j L} \equiv(2 \pi \hbar)^{3} f_{j L} / 2$ is the occupation number that describes the distribution of the photon-lepton plasma in the early universe. It was recently shown [19] that the collision term of the Boltzmann equation for dark matter kinetically coupled to the primordial lepton-photon plasma can be generically written in the form of the Fokker-Planck operator:

$$
C\left[f_{\chi}\right]=\gamma \frac{\partial}{\partial \mathbf{p}} \cdot\left[\left(\mathbf{p}-m_{\chi} \mathbf{v}_{L}\right) f+m_{\chi} T_{L} \frac{\partial f}{\partial \mathbf{p}}\right],
$$

where $\gamma$ is the rate coefficient that incorporates all the contributions of a particular particle physics model into this problem and determines the temperature of kinetic decoupling. A larger rate coefficient leads to a lower temperature and hence later time for kinetic decoupling. In turn, the later time of decoupling leads to a greater suppression of the power on the small scales and therefore reduces the number of small scale dark matter halos. The rate coefficient provides an important link between a given particle physics model and the growth of dark matter perturbations in the early universe and can possibly lead to information on particle physics models from astrophysical observation of the universal structure. 
We can calculate the interaction rate by performing the integration of the collision term of Boltzmann equation 3.1 with scattering amplitude $\mathcal{M}$ calculated within the particular particle physics model. The main scattering channels determining the kinetic coupling of dark matter to the primordial plasma in the Littlest Higgs with Tparity model are presented in Fig. 3.1. After lengthy calculations, the details of which will be presented elsewhere, the rate coefficient in the Littlest Higgs with T-parity model can be written as:

$$
\gamma=C_{L H T} \frac{T^{4}}{M_{A_{h}}^{3}}
$$

Here $C_{L H T}$ is a numerical coefficient corresponding to a particular set of parameters of the model.
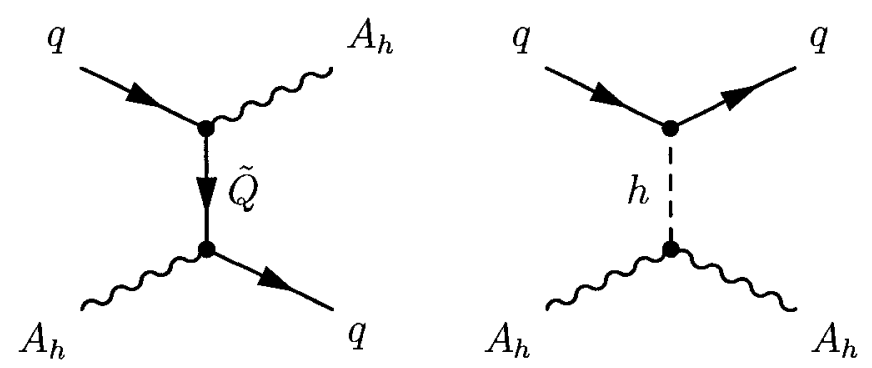

Figure 3.1: Dominant channels for the Littlest Higgs with T-parity dark matter scattering with SM particles in the primordial plasma. This scattering maintains dark matter in kinetic equilibrium with the surrounding cosmic plasma long after freeze-out and determines the formation of the smallest dark matter halos.

\subsection{Transfer function and prospective research}

Prior to kinetic decoupling, dark matter is tightly coupled to the primordial leptonphoton plasma and shares the same pressure-induced oscillations that lead to acoustic peaks in the cosmic microwave background (CMB). This leads to small-scale oscil- 
lations in the power spectrum of density fluctuations [20] and also produces overall suppression of power on small scales. In order to obtain the full picture of the behavior of dark matter during the period of kinetic coupling and to construct the power spectrum of the resulting dark matter fluctuations we need to solve the Boltzmann equation 3.1 including first order perturbations. To address this problem we have written a code that solves the coupled Boltzmann equations for a particular period of kinetic coupling and prepares the transfer functions needed for cosmological simulations that can trace the development of structure beyond the regime of linear perturbations. The resulting transfer functions for the case of the Littlest Higgs dark matter are presented in Fig. 3.2. The transfer function that is obtained in the CDM model is also plotted.

The analysis of the structure formation opens a whole new area of constraints and checks that can elucidate the direction for theories beyond the Standard Model. Combining increased computational capabilities with the advanced observational facilities enables a direct test of the particle physics models. By implementing the transfer functions obtained into the N-body simulations, we are able, for instance, to unambiguously predict properties of small-scale dark matter clumps. Such clumps can be detectable through $\gamma$-ray production from particle annihilation [53], through fluctuations in the event rate of direct detection experiments, through their tidal gravitational effect on wide orbits of objects near the outer edge of the solar system, or through variations in the pulsar timing rates [54]. 


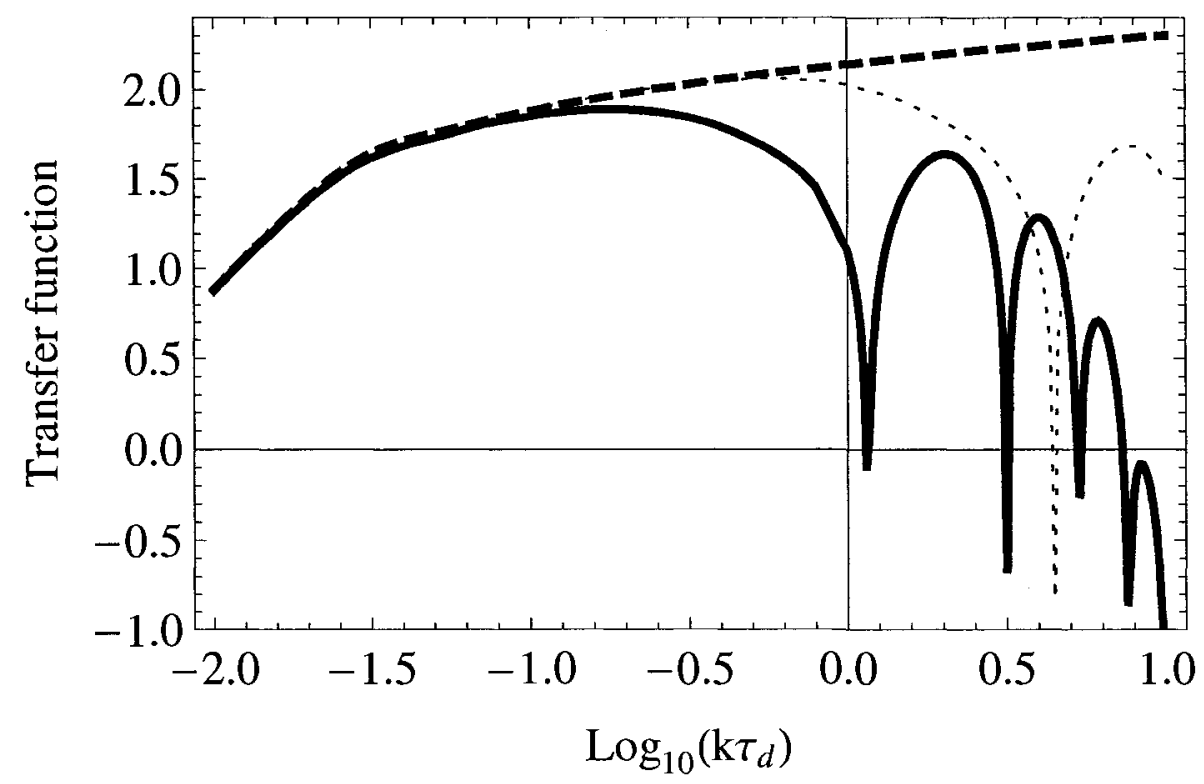

Figure 3.2: Transfer function for the Littlest Higgs dark matter perturbations after the period of kinetic decoupling. The solid (dotted) line corresponds to $M_{A_{h}}=100$ $\mathrm{GeV}\left(M_{A_{h}}=300 \mathrm{GeV}\right)$. Other model parameters are chosen to be consistent with relic density constraints. The dashed line shows the transfer function in the CDM cosmology [19]. 


\section{Chapter 4}

\section{Collider phenomenology}

As we have shown in the previous sections, astrophysical observations provide stringent constraints on the properties of dark matter particles and, hence, can direct the search for dark matter at existing and future colliders. Astrophysical probes, however, are unable to determine and study the exact properties of a dark matter particle and it largely remains for collider physics to unravel the mystery surrounding dark matter.

Regardless of its origin, if cold dark matter is composed of WIMPs, then it may be possible to produce and study the dark matter particle(s) directly at the LHC. In fact, the LHC may well turn out to be a dark matter factory, where the nature of dark matter particles and their properties might be studied in a controlled environment [55]. In any collider experiment, WIMPS would be like neutrinos in that they would escape the detector without depositing any energy in the experimental apparatus, resulting in an apparent imbalance of energy and momentum in collider

events. While WIMPs would manifest themselves only as missing (transverse) energy at collider experiments, it should nevertheless be possible to analyze the visible par- 
ticles produced in WIMP-related processes to study the new physics associated with the WIMP sector. This is particularly true for Little Higgs dark matter because of the well defined signatures that are strongly related to the symmetry breaking scale $f$ as well as to the mass of dark matter particles.

\subsection{Dark matter and missing transverse energy}

Before discussing the collider phenomenology of dark matter it is important to clearly formulate questions and objectives of such a discussion. First of all we need to emphasize that, at present, there is no direct accelerator evidence for the existence of new physics beyond the Standard Model and, in particular, no indication of the Little Higgs mechanism. Furthermore, there is no direct confirmation of the existence of a particle or particles that account for the observed dark matter phenomena. The combined effort of theoretical and experimental cosmology and high energy physics has lead to these ideas and strongly favors them, but they are still unproven ideas. So we can formulate two closely related initial questions for the LHC that are of interest to both hep- and astro-communities: do we observe physics that deviates from SM predictions and do we observe effects that can be unambiguously attributed to dark matter?

The first of these questions is much more general and requires no knowledge of the physics beyond the SM. All that is needed is to compare the SM predictions with results from the LHC. On the contrary, the second question cannot be approached in a model independent way and will require analysis of the full spectrum of LHC observations. As we discussed in the introductory part of this thesis there is strong astrophysical evidence that dark matter is comprised of WIMPs - non-baryonic, heavy 
particles that do not carry either electric or color charge. The weakness of dark matter interaction with the SM particles precludes its direct observation in the detectors of hadron colliders such as the Tevatron and LHC. Dark matter can manifest itself only through missing energy - an apparent imbalance of energy and momentum in a particular event. Because the interacting partons (quarks and gluons) have only a fraction of the proton's energy that is not known on an event-by-event basis, we must focus on kinematic quantities transverse to the beam direction. Momentum conservation requires that the sum of all momenta transverse to the beam direction is zero. If something invisible is produced (such as a WIMP or neutrino), then the vector sum of all the transverse momenta will not be zero, but rather the opposite of the momentum of the undetected particle(s). Therefore, by measuring energy deposited in each calorimeter cell of a detector we can determine the missing energy vector defined as (see for example [56])

$$
\mathbf{E}_{T}^{m i s s}=-\sum\left(\frac{E_{n} \cos \left(\phi_{n}\right)}{\cosh \left(\eta_{n}\right)} \hat{\mathbf{x}}+\frac{E_{n} \sin \left(\phi_{n}\right)}{\cosh \left(\eta_{n}\right)} \hat{\mathbf{y}}\right)=E_{x}^{m i s s} \hat{\mathbf{x}}+E_{y}^{m i s s} \hat{\mathbf{y}}
$$

where $E_{n}$ is the total energy deposited in a calorimeter cell characterized by azimuthal angle $\phi_{n}$ and pseudorapidity $\eta_{n}$, which specifies the angle $\theta$ with respect to the beam direction

$$
\eta=-\ln \left[\tan \left(\frac{\theta}{2}\right)\right]
$$

Unfortunately, dark matter is not the only source of missing energy in collider experiments. Other sources that can produce an apparent imbalance of transverse energy and momentum are:

- losses of energy in uninstrumented regions of the detector ("cracks")

- additional energy unrelated to the primary interaction (cosmic rays) 
- limited calorimeter resolution on the energy of jets

- neutrinos and also long lived neutral kaons and neutrons

For the analysis of dark matter it is imperative to correctly account for all sources of missing energy. Topological defects like uninstrumented regions, effects of calorimeter resolution, and side sources like cosmic rays are studied in great detail during the preparation phase of any collider experiment (see for example [57]). From the theoretical side we can only predict and subtract the missing energy background caused by neutrinos that are produced in the SM processes. Neutrinos can also be produced in the events that involve new heavy particles. For instance the new heavy $Z_{h}$ boson in the Simplest Little Higgs with T-parity model can invisibly decay either into two dark matter particles or into two ordinary neutrinos (see Fig. B1). This type of background can not be predicted in advance and strongly complicates the investigation of dark matter at colliders.

So the first question of the dark matter study at the LHC will be to confirm that there are missing energy events that exceed the SM prediction on a statistically significant level. The next step requires analysis of data from various independent channels to determine what is the favored extension of the SM and what are the possible alternatives. After our attention is constrained to a limited number of models with somewhat constrained parameters we can start matching the observed distributions of the missing energy and related SM particles with the predictions of those models.

In this thesis, we show that there is a significant excess of missing energy events for large regions of the allowed parameter space in both the Littlest Higgs and Simplest Little Higgs with T-parity models. We further analyse the signatures 
produced by dark matter in the SLHT model and comment on the dark matter signal from the LHT.

\subsection{MadGraph and calculation methods}

Calculations of the scattering cross sections as well as simulations of both dark matter production and the SM background processes at the LHC were performed with the MadGraph package [58]. MadGraph is a Monte-Carlo event generator that generates amplitudes and events for any allowed process (with up to 9 external particles) in a large variety of models including models implemented by the user. It also automatically includes parton distribution functions (PDF) for protons and antiprotons which allows simulations of events at both the LHC and the Tevatron.

Parton distribution functions allow us to obtain cross sections of highly energetic hadronic collisions by calculating the cross sections for individual parton level processes [59]. An incoming hadron of momentum $p_{h}$ is represented by partons $i$ carrying longitudinal momentum fractions $x_{i}\left(0 \leq x_{i} \leq 1\right)$. Transverse momenta of partons are neglected. The total cross section can be obtained by the following integral

$\sigma(A B \rightarrow c X)=\sum_{a, b} C_{a b} \int d x_{a} d x_{b}\left[f_{a / A}\left(x_{a}\right) f_{b / B}\left(x_{b}\right)+(A \leftrightarrow B\right.$ if $\left.a \neq b)\right] \hat{\sigma}(a b \rightarrow c X)$.

Here $A$ and $B$ are the incidents hadrons, $c$ and $X$ are final state particles, $f_{a / A}$ is a parton density of $a$ in $A, C_{a b}$ - color-averaging factors, and $\hat{\sigma}$ is a parton level cross section summed over initial and final colors. For all calculations in this work we used the CTEQ6L1 (Coordinated Theoretical Experimental Project on QCD) PDF package [60]. 
For the purpose of our analysis we have introduced the Simplest Little Higgs with T-parity (SLHT) model into the MadGraph. For the convenience of future studies we summarize the Feynman rules specific to the SLHT model in Appendix A of this thesis. We also implemented the dark matter relevant sector of the Littlest Higgs with T-parity model into MadGraph and checked the consistency of our results with the previously realized implementations of the LHT in MadGraph [61] and CalcHEP [49].

MadGraph integrates a large chain of tools that allow the user to go directly from a given physics model to simulated events in a detector. Invariant amplitudes are calculated using HELAS [62] - helicity amplitude subroutines for Feynman diagram evaluations, which allows fast calculation of a large number of diagrams. Simulation of detector events can be performed with the PGS/PYTHIA [63] package that accounts for specific characteristics of detectors and produces events similar to those expected in the actual experiments like ATLAS or CMS at the LHC. Computations of the MadGraph processes can be done both online on the web server provided by the MadGraph team and offline using either a single CPU or a large cluster. MadGraph also contains the MadAnalysis plotting library that can create histograms of various kinematic quantities (transverse momenta and energies, rapidity, invariant mass, etc.) from Les Houches and LHC Olympics events in Topdrawer format [64].

For the purpose of dark matter simulations in the Simplest Little Higgs model we have chosen 3 sets of parameters, consistent with the relic density constraints that are discussed in Chapter 5. For the generation of LHC events, standard cuts were imposed: minimum transverse momentum of a jet event $p t_{j e t}^{m i n}=20 \mathrm{GeV}$, maximum rapidity of a jet $\eta_{j e t}^{\max }=2.5$. Also, for all collider calculations we use the standard set of major SM parameters [39]: $G_{F}=1.16637(1) \times 10^{-5} \mathrm{GeV}^{-2}-$ Fermi constant, 
$\alpha=1 / 137.036(1)$ - fine-structure constant, $\sin \theta_{W}^{2}\left(M_{Z}\right)=0.23152(14)$ - square of the electroweak mixing angle, and $\alpha_{s}\left(M_{Z}\right)=0.1176(20)$ - strong coupling constant.

Finally, we would like to emphasize that MadGraph is only one representative of a family of programs that perform event generation and collider simulations. Others include, but are not limited to, CompHEP/CalcHEP [65, 66], SHERPA [67] and WHIZARD [68]. A major advantage of MadGraph over CalcHEP is the ability to generate matrix elements for a large number of final state particles, whereas CalcHEP is limited to 2 particles in the final state. A significant advantage of MadGraph over WHIZARD, that eventually determined our choice, is a simple and very well documented procedure of implementing models of new physics.

\subsection{LHC signatures of Little Higgs dark matter}

LHC phenomenology of the Littlest Higgs dark matter has received a lot of attention in the literature $[16,46,48,49]$. It has been found that the most promising channels of dark matter production are

$$
\begin{gathered}
p p \rightarrow W_{h}^{+} W_{h}^{-} \rightarrow W^{+} W^{-} A_{h} A_{h}, \\
p p \rightarrow W_{h}^{ \pm} Z_{h} \rightarrow W^{ \pm} h A_{h} A_{h}, \\
p p \rightarrow W_{h}^{ \pm} A_{h} \rightarrow W^{ \pm} A_{h} A_{h},
\end{gathered}
$$

Feynman diagrams contributing to these channels along with the SM background processes are presented in Figs. 4.1, 4.2, and 4.3 respectively. Also, as pointed out in [48], if new heavy quarks have relatively low masses, close to the mass of the dark 
matter particle, characteristic monojet and dijet plus missing energy signatures can be expected. In these processes, dark matter can be produced in association with new heavy quarks which subsequently decay into the Standard Model quarks and produce jets after hadronisation.

The typical feature of the dark matter production events is that the distribution of missing $E_{T}$, as well as $p_{T}$ distributions of the observed particles, is shifted toward higher energies in comparison with SM processes. For instance in Figs. 5.11 and 5.12 of Chapter 5 we show examples of the distributions of the transverse missing energy for $A_{h}$ production in association with one and two W-bosons respectively. The observed distributions clearly stand above the SM background at large values of Missing $E_{T}$. However, it is important to note that W-bosons are observed at the LHC only through their decay products i.e. either quarks that form jets or leptons plus neutrinos. This significantly complicates the analysis of dark matter production and for a large region of parameter space makes the detection impossible [16]. In order to observe dark matter production in these processes it is necessary to impose kinematic cuts on the observed leptons, which generally increases the signal to background ratio. A specific channel of W-boson decays, namely $p p \rightarrow W_{h}^{+} W_{h}^{-} \rightarrow W^{-} A_{h} W^{+} A_{h} \rightarrow \mu^{+} e^{-} \bar{\nu}_{e} \nu_{\mu} A_{h} A_{h}$, and kinematic cuts for the heavy photon production in this channel were discussed in [46] and it was shown that heavy photon detection in this channel is possible over a large region of LHT parameters.

In this thesis we focused on one Simplest Little Higgs dark matter candidate - a heavy neutrino. We identify two promising channels of dark matter production:

$$
\begin{gathered}
p p \rightarrow j e t+N \bar{N}, \\
p p \rightarrow l^{+} l^{-}+N \bar{N},
\end{gathered}
$$


where $l^{+} l^{-}$represents either $e^{+} e^{-}$or $\mu^{+} \mu^{-}$pair. Feynman diagrams contributing to these channels are presented in Figs. 4.4 and 4.5, respectively. Cross sections at LHC energy as a function of dark matter mass for these two channels are shown in Fig. 4.7. As can be seen from the Feynman diagrams in Fig. 4.4, monojet associated dark matter production is enhanced by the QCD coupling and we can expect a large number of events for this channel. A large cross section of the monojet plus missing energy channel makes it an extremely important signature of the Simplest Little Higgs with T-parity model. This signature may help to distinguish this model from both supersymmetry, in which dark matter is predominantly produced in multijet events, and the Littlest Higgs with T-parity model, in which monojet dark matter production is highly suppressed. In fact we identify monojet associated dark matter production as a smoking-gun signature of the Simplest Little Higgs with T-parity. If the SLHT is realized in nature then this process should be observed at a very early stage of the LHC operation and the model can be confirmed with the dilepton channel which has a distinct signature, but a lower cross section.

The integrated luminosity of the LHC is expected to be around $10 \mathrm{fb}^{-1}$ after the first year of operation and more than $300 \mathrm{fb}^{-1}$ over the full period of operation. From Fig. 4.7 we can see that, if the Simplest Little Higgs mechanism is realized in nature, and the heavy neutrino is light enough, then dark matter indeed will be copiously produced at the LHC. However, the SM model background is also significant. Some Feynman diagrams contributing to the SM background of the monojet and dilepton channels are presented in Fig. 4.6. The total cross section at LHC for the monojet plus missing energy production in the SM is $\sigma \approx 2 \cdot 10^{3} p b$, while for the dilepton plus missing energy channel $\sigma \approx 2 p b$. In both cases we expect many more background events than events with dark matter production. However, because of the 
large mass of the dark matter particle, signal events appear in the kinematic regions where the SM background is relatively small. In Chapter 5 we show the predicted signal of the missing energy events compared to the SM background for various values of the dark matter mass that clearly show the possibility of $5 \sigma$ detection for the considered region of SLHT parameter space. Here we also need to mention that, along with the two neutrino background shown in Fig. 4.6, there are processes containing four neutrinos; however, our calculations show that the cross section of such events is negligible in both channels and hence their contribution can be safely neglected.

To conclude this section we note that over the last years there has been an intensive effort to detect dark matter production at the Tevatron. However, this effort did not yield any positive results, which shows that dark matter production lies beyond the kinematic regions probed in proton antiproton collisions at $s=\sqrt{2} \mathrm{TeV}$. In Fig. 4.8 we show the cross sections for the same SLHT dark matter production at the Tevatron energies. We clearly see that the dilepton channel lies beyond reach of the Tevatron for all values of the analyzed parameters. Dark matter associated events in the monojet channel also can not be observed in the kinematic region accessible to the Tevatron because of the large SM background $\sigma_{S M}=140 p b \gg \sigma_{D M}$ for all considered values of the SLHT parameters. 

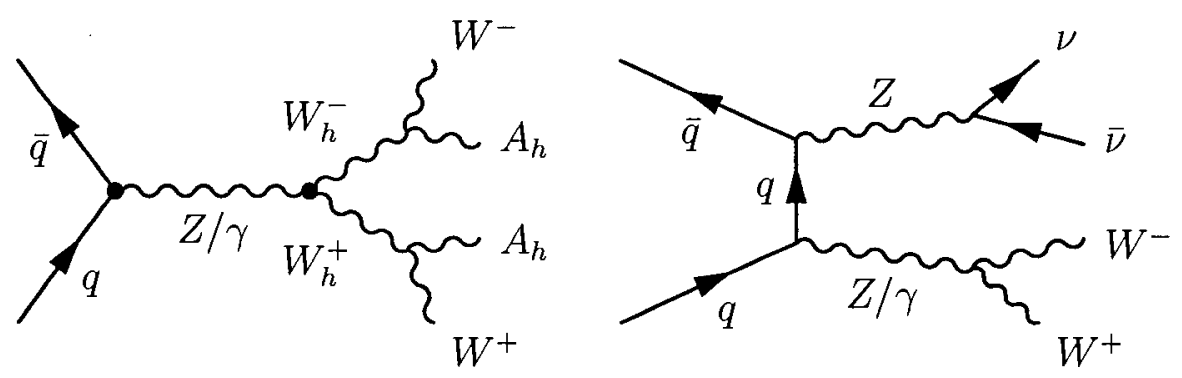

Figure 4.1: Sample Feynman diagrams for $p p \rightarrow W_{h}^{+} W_{h}^{-} \rightarrow W^{+} W^{-} A_{h} A_{h}$ in the Littlest Higgs with T-parity model (left) and SM background (right).
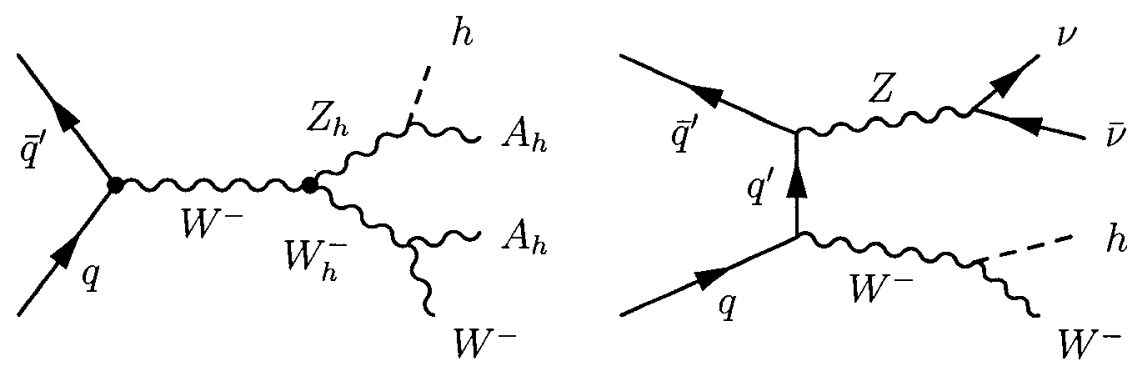

Figure 4.2: Sample Feynman diagrams for $p p \rightarrow W_{h}^{ \pm} Z_{h} \rightarrow W^{ \pm} h A_{h} A_{h}$ in the Littlest Higgs with T-parity model (left) and SM background (right).
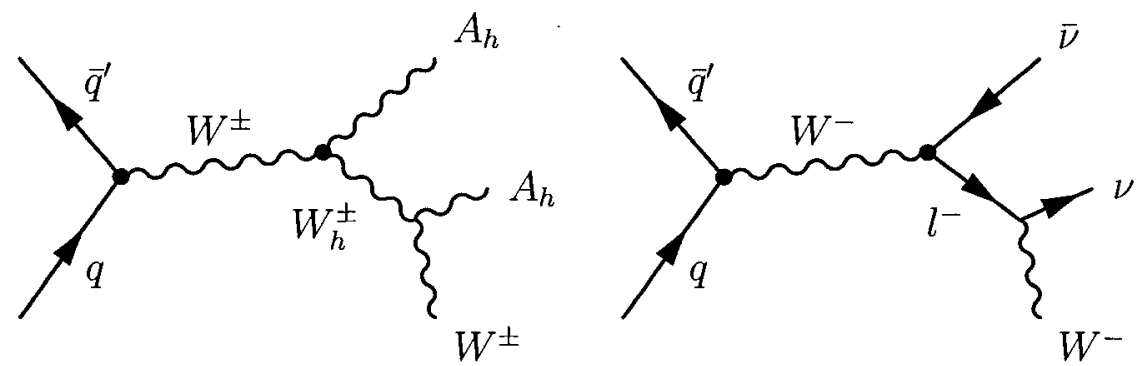

Figure 4.3: Sample Feynman diagrams for the $W$ associated dark matter production at the LHC in the Littlest Higgs with T-parity model (left) and the SM background for this process (right). 

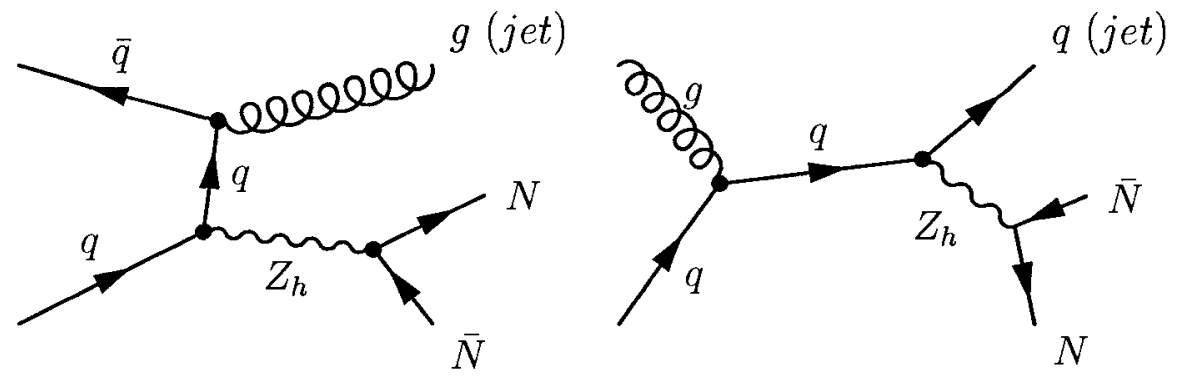

Figure 4.4: Sample Feynman diagrams for the monojet associated dark matter production at the LHC in the Simplest Little Higgs with T-parity model.
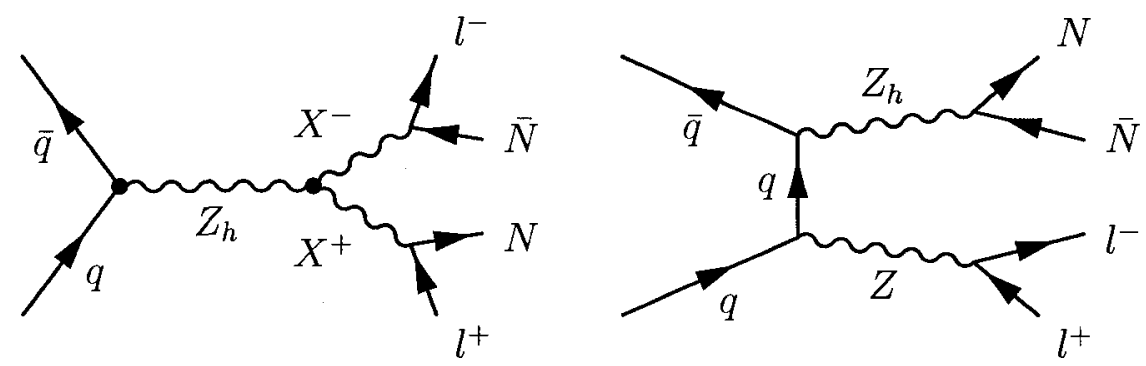

Figure 4.5: Sample Feynman diagrams for the dileptonic dark matter production at the LHC in the Simplest Little Higgs with T-parity model.
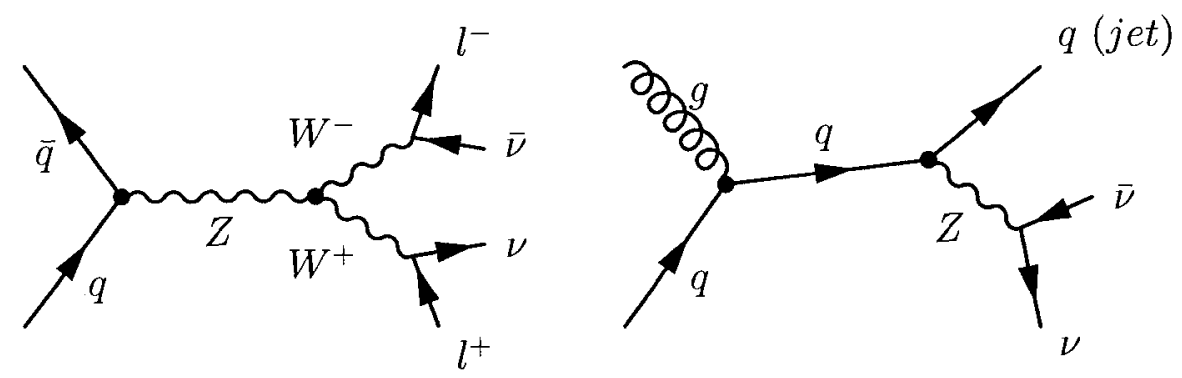

Figure 4.6: Sample Feynman diagrams for the Standard Model background to the dileptonic (left) and monojet (right) associated dark matter production at the LHC. 


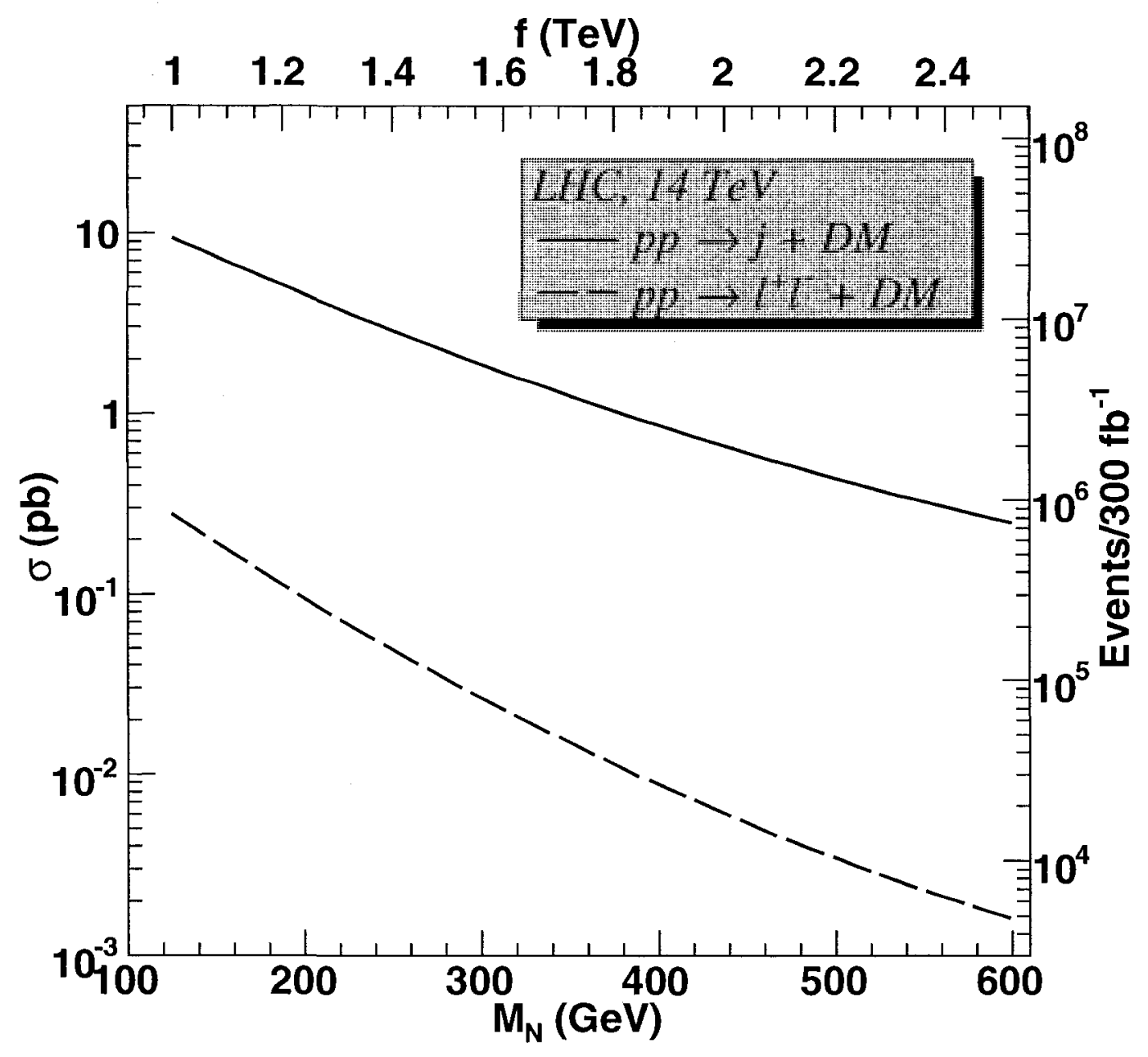

Figure 4.7: Cross section of dark matter production at the LHC is plotted as a function of dark matter mass. On the top scale we identify the SLHT symmetry breaking scale $f$ that corresponds to the dark matter mass via the relic density constraints of Eq. 5.1. The number of events for an integrated luminosity of $300 \mathrm{fb}^{-1}$ is plotted on the second $y$-axis. 


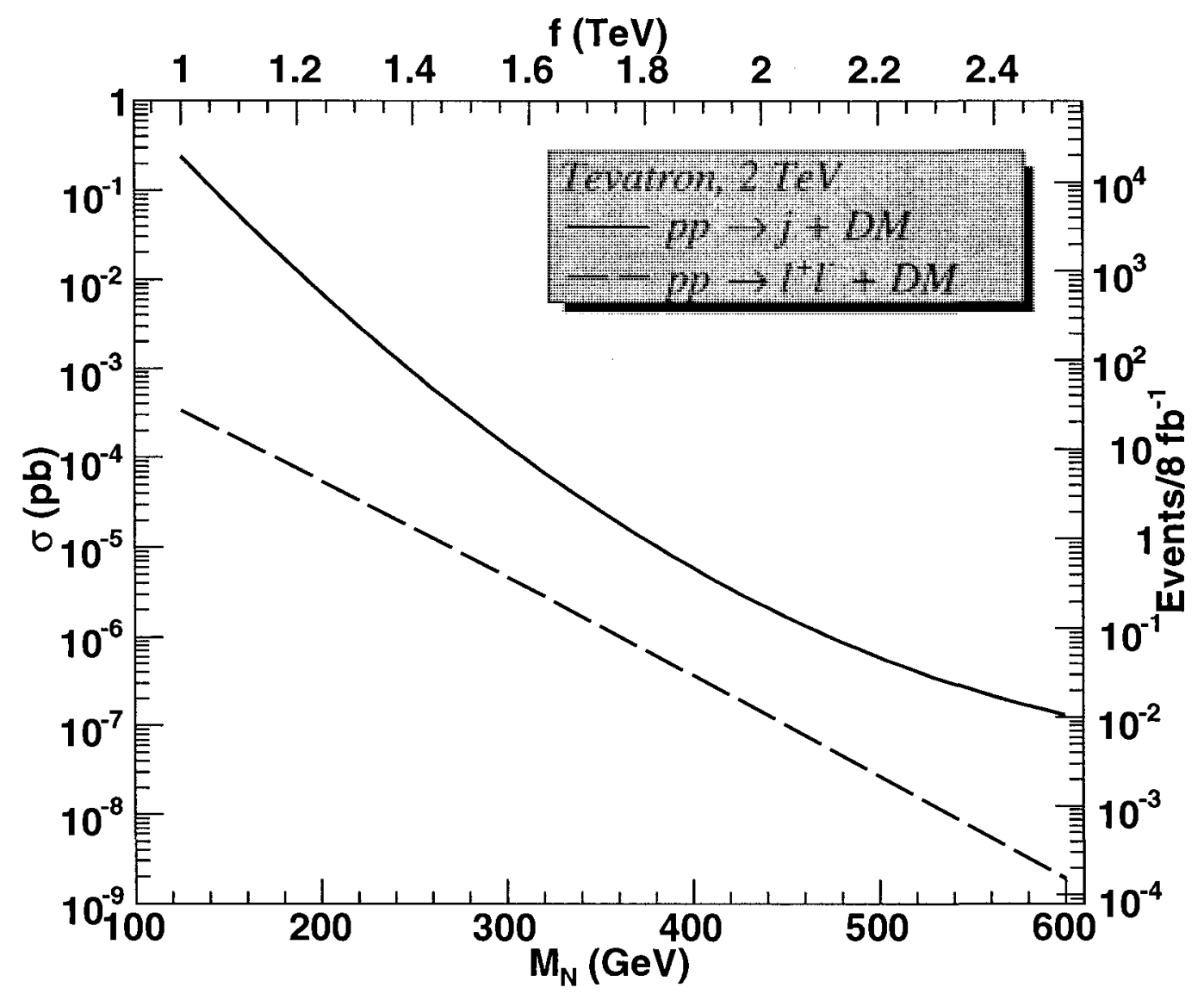

Figure 4.8: Cross section for dark matter production at the Tevatron in the Simplest Little Higgs Model. On the top scale we identify the SLHT symmetry breaking scale $f$ that corresponds to the dark matter mass via the relic density constraints of Eq. 5.1. The number of events for $8 \mathrm{fb}^{-1}$ is plotted on the second $\mathrm{y}$-axis. 


\section{Chapter 5}

\section{Results}

Fig. 5.1 shows constraints that are imposed on the parameter space of the Simplest Little Higgs with T-parity model from the relic density calculations. For the calculation of the relic density constraints we wrote a Mathematica [69] code using the Feyncalc package [70] to calculate invariant amplitudes of the annihilation processes discussed in Section 2.2. We plot allowed regions that fall within $2 \sigma$ and $5 \sigma$ around the result obtained by the WMAP team $0.094<\Omega_{D M} h^{2}<0.126$ [2]. The allowed region can be conveniently parametrized by the linear relation that links dark matter mass and the symmetry breaking scale of the initial global symmetry in the SLHT:

$$
f=3.2 M_{N}+600,
$$

where $f$ and $M_{N}$ are in $\mathrm{GeV}$ and the mass of the Higgs boson is fixed to be $M_{h}=120$ $\mathrm{GeV}$. Using the above constraints we chose three sets of parameters for the analysis of collider phenomenology of the SLHT dark matter. The parameters that we chose are given in Table 5. They cover most of the allowed parameter space and hence are representative of the model in general. Calculation of the SLHT parameters is discussed in Appendix B. 


\begin{tabular}{|c||c|c|c|}
\hline Parameter in GeV & Set 1 & Set 2 & Set 3 \\
\hline $\mathrm{f}$ & 1000 & 1560 & 2520 \\
\hline$M_{N}$ & 125 & 300 & 600 \\
\hline$M_{Z_{h}}$ & 560 & 872 & 1409 \\
\hline$M_{h}$ & 120 & 120 & 120 \\
\hline$\Gamma_{Z_{h}}$ & 4.0 & 5.8 & 8.6 \\
\hline$M_{X}$ & 460 & 717 & 1158 \\
\hline$\Gamma_{X}$ & 1.1 & 1.5 & 2.0 \\
\hline$M_{T}$ & 983 & 1533 & 2477 \\
\hline$M_{S}, M_{N_{2}}, M_{N_{3}}$ & 707 & 1103 & 1782 \\
\hline
\end{tabular}

Table 5.1: Parameters used for the analysis of dark matter production at the LHC in the Simplest Little Higgs with T-parity model. Values of the tested parameters are chosen to be consistent with the relic density constraints from Fig. 5.1 and to cover most of the allowed parameter space. 


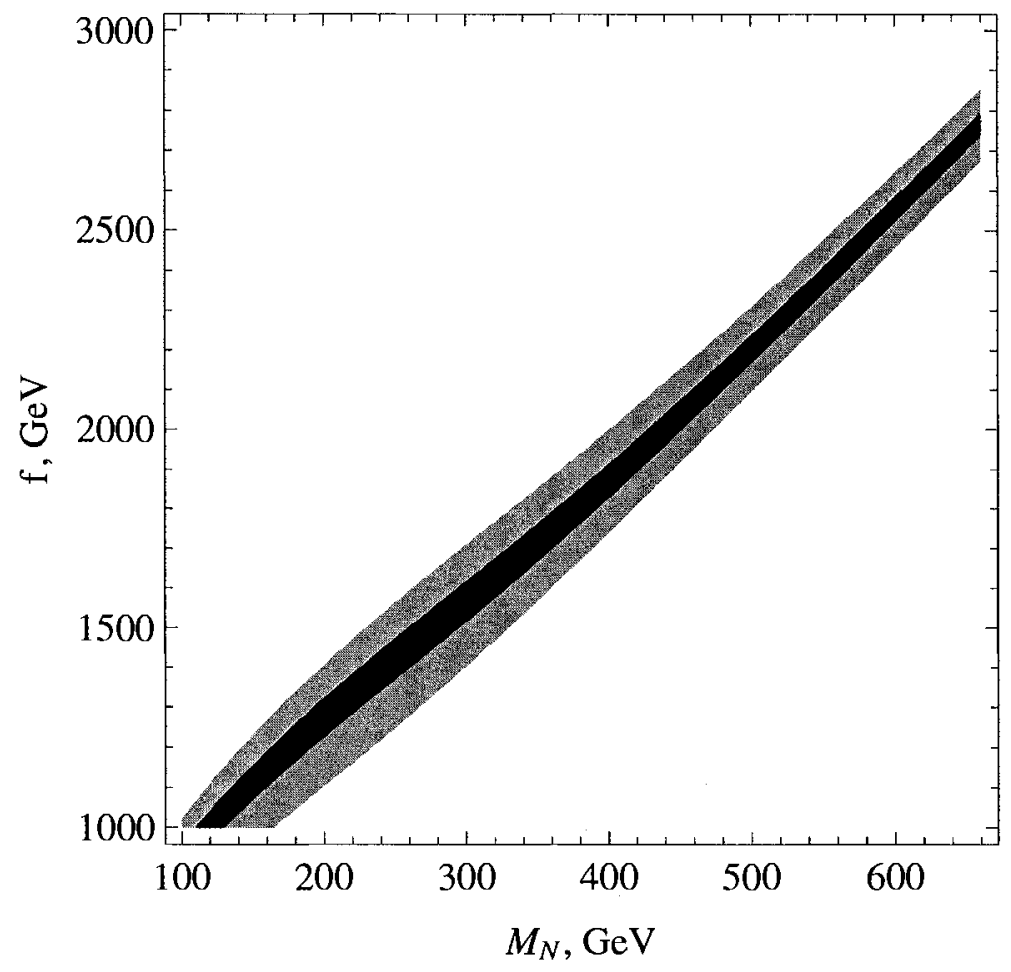

Figure 5.1: Relic abundance constraints on the parameter space of the Simplest Little Higgs with T-parity model. On this plot the dark region corresponds to $2 \sigma$ variation about the WMAP observed relic density, while the gray region covers $5 \sigma$ confidence level. 
Fig. 5.2 shows the expected distribution of the missing transverse energy for the monojet plus missing energy production in the Simplest Little Higgs with Tparity model at the LHC for an integrated luminosity of $L=300 \mathrm{fb}^{-1}$. This plot was generated using our implementation of the SLHT model in the MadGraph event generator for the parameter Set 1 of Table 5. The SM prediction for the missing energy distribution is also shown. We see a significant excess of events with large values of missing energy in the model with dark matter. The greatest excess is observed around the mass of the heavy neutral gauge boson $Z_{h}$ that can invisibly decay into either neutrinos or dark matter particles with the branching ratios presented in Fig. B1 of Appendix B.

A similar plot is presented in Fig. 5.3 for the dilepton plus missing energy production. In this case the signal shows a significant excess over the background in the regions where the SM is almost negligible. However, the expected number of events is also much smaller than in the monojet case.

The evolution of dark matter production signals (without SM background) with the increasing mass of dark matter particles and the corresponding increase of other masses in the model is shown in Fig. 5.4 for the monojet channel and in Fig. 5.5 for the dilepton channel. We clearly see the decrease in the signal with the increasing mass of the dark matter particle. For masses $M_{N} \gtrsim 600 \mathrm{GeV}$ the signal in the dilepton channel becomes indistinguishable from the statistical background, however dark matter can still be detected through the monojet channel.

In order to increase the signal to background ratio we need to impose kinematic cuts on the observed particles as well as on the missing energy events. To determine optimal cuts we considered predicted distributions for the observed particles as well as for missing energy in both the SLHT and the SM. In this study we analyze cuts only for 
the dilepton channel, since the monojet signal is very well distinguished over the SM background without introducing any cuts over all values of the considered parameters. In Figs. 5.6, 5.7, and 5.8 we plot expected distributions of the electron's transverse momentum $p_{T}$, electron's rapidity, and invariant mass of the electron positron pairs for dilepton associated dark matter production and compare those distributions with the SM expectation. Clearly electrons produced in dark matter related processes are more energetic and mainly appear in the central region of the detector. This suggests the introduction of a variety of kinematic cuts depending on the specific objectives. In this thesis we considered a cut on the charged lepton momentum $p_{T}$ which allows us to significantly increase the threshold on the dark matter mass that can be observed in the dilepton channel. In Fig. 5.9 we show the dilepton plus missing energy signal for the second parameter set (i.e. for the heavy neutrino mass of $M_{N}=300 \mathrm{GeV}$ ) both before and after kinematic cuts. This cut reduces the cross section of the SM background more than 3 orders in magnitude, while the signal is decreased only 2 orders. Remarkably, this cut also allows us to study a different aspect of the SLHT model, namely the mass of the new heavy neutral gauge boson $Z_{h}$, with the observation of the invariant mass distribution for the charged leptons. In Fig. 5.10 we clearly see that the distribution in the SLHT model has a distinctive peak around the mass of $Z_{h}$.

Figs. 5.11 and 5.12 show expected distributions of the missing transverse energy in $p p \rightarrow W^{ \pm}+$Missing $E_{T}$ and $p p \rightarrow W^{+} W^{-}+$Missing $E_{T}$ channels in the Littlest Higgs with T-parity model. These distributions were obtained using MadGraph, for the dark matter mass $M_{A_{h}}=110 \mathrm{GeV}$ (corresponding to the symmetry breaking scale $f=700 \mathrm{GeV}$ ) and Higgs boson mass determined from the first expression of Eq. 2.8. 


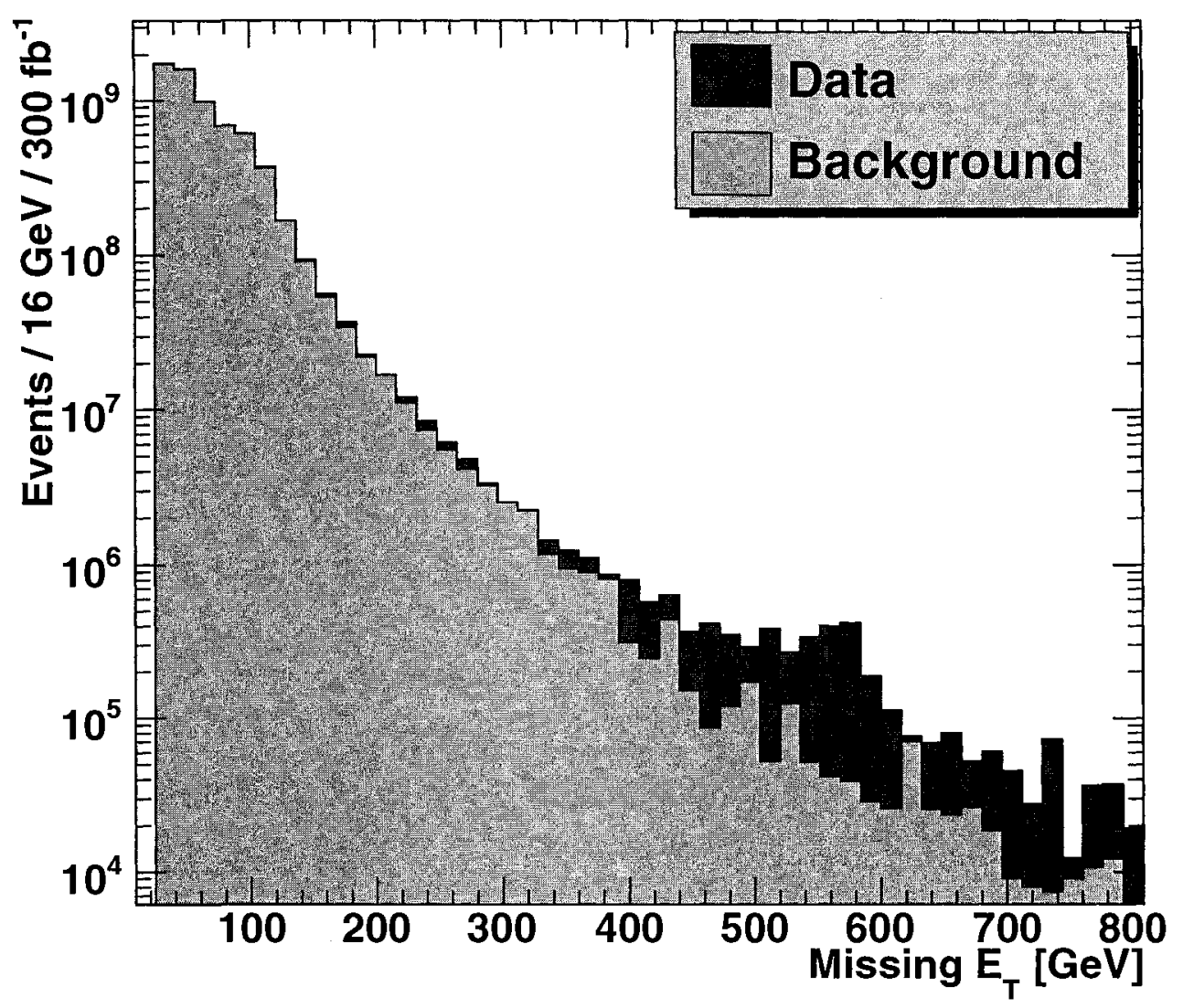

Figure 5.2: Missing transverse energy distribution for the monojet plus missing energy production in the Simplest Little Higgs with T-parity model at the LHC and the SM background. This plot was generated with MadGraph using set 1 model parameters of Table 5, for the integrated luminosity of $300 \mathrm{fb}^{-1}$. 


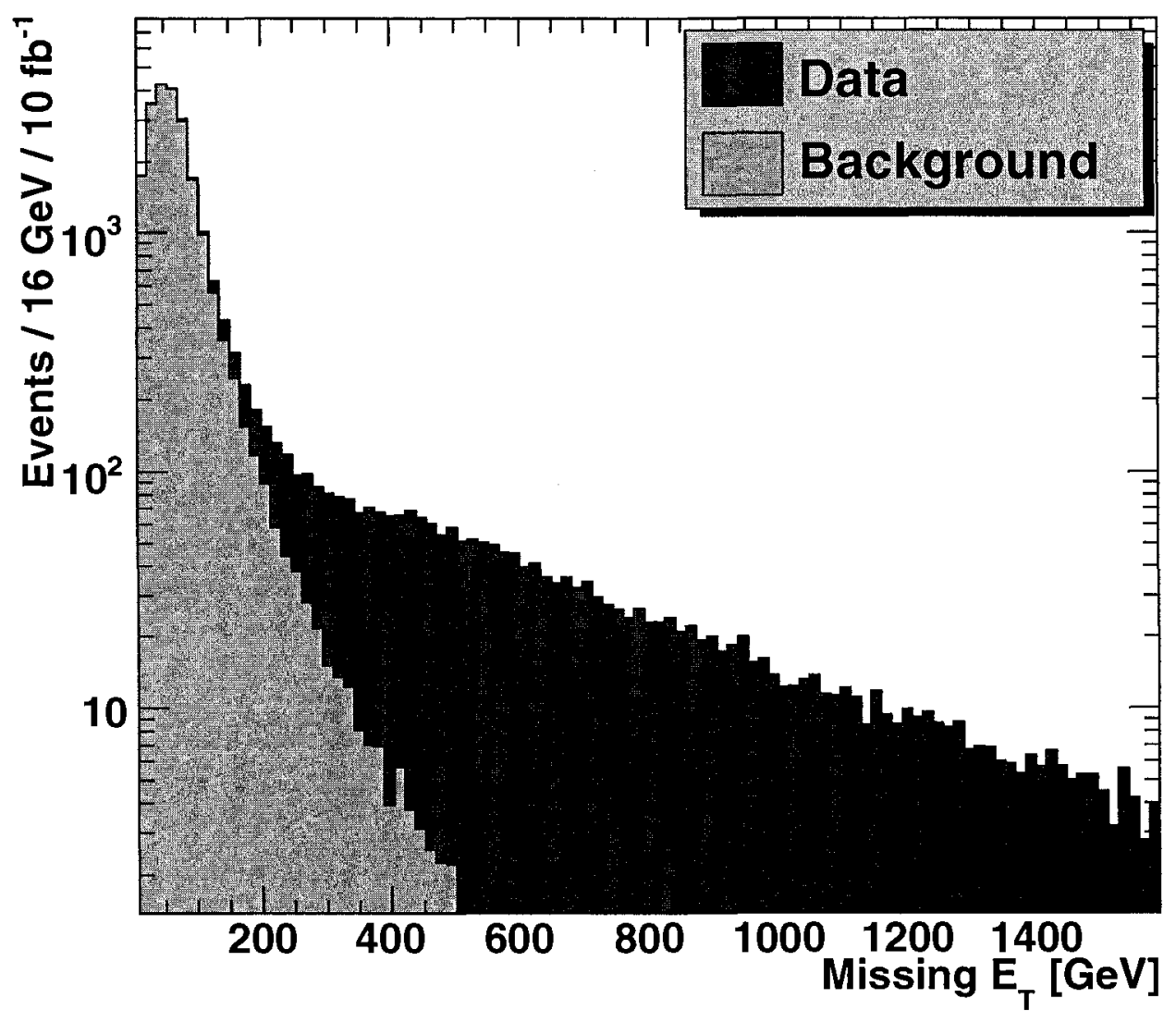

Figure 5.3: Missing transverse energy distribution for the dilepton plus missing energy production in the Simplest Little Higgs with T-parity model at the LHC and the SM background. This plot was generated with MadGraph using set 1 model parameters of Table 5, for the integrated luminosity of $10 \mathrm{fb}^{-1}$. 


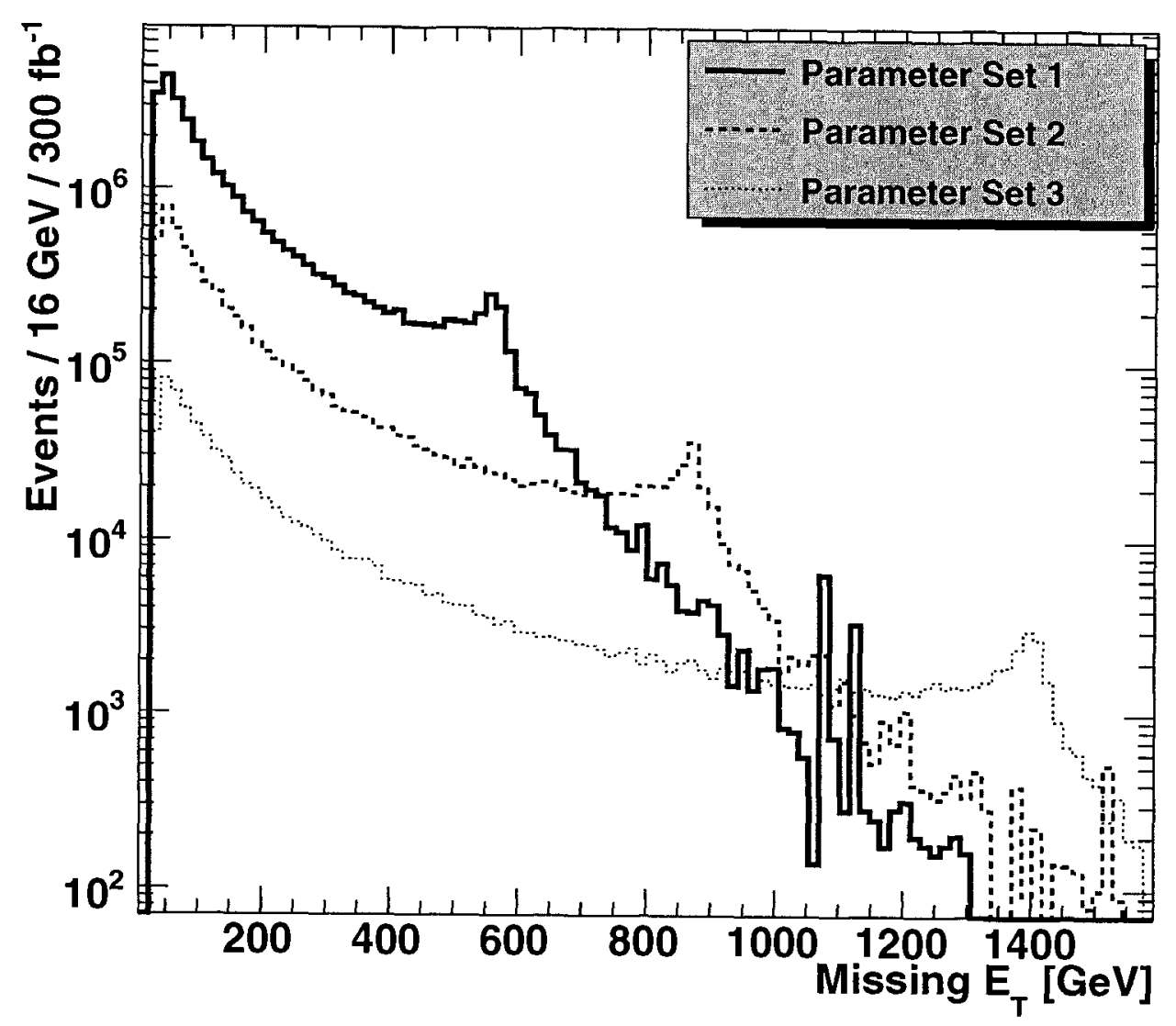

Figure 5.4: Comparison of the missing transverse energy distribution for the monojet associated dark matter production in the SLHT model at the LHC for the 3 parameter sets of Table 5. The integrated luminosity is $300 \mathrm{fb}^{-1}$. Each distribution clearly exhibits a peak around the corresponding mass of $Z_{h}$. 


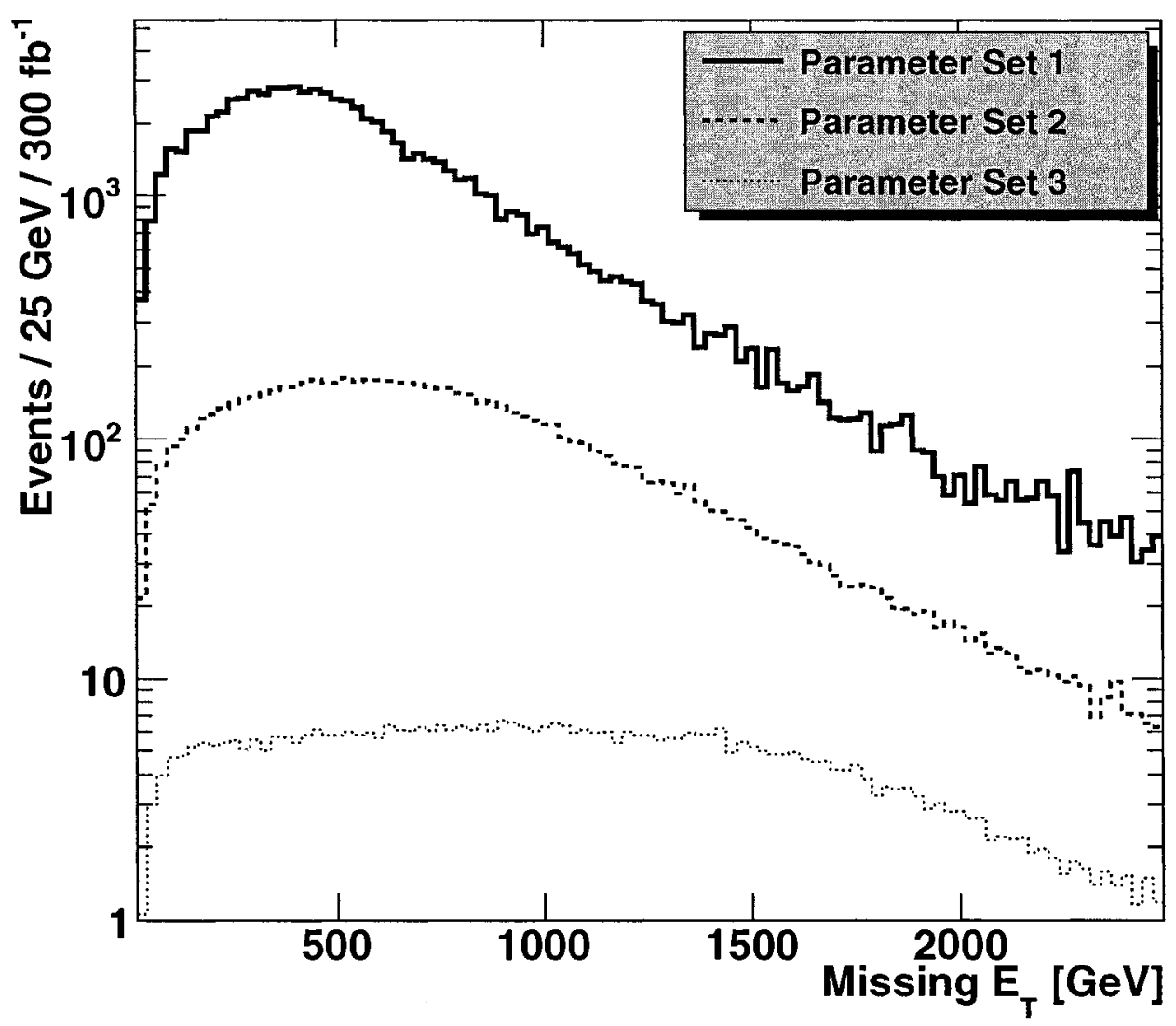

Figure 5.5: Comparison of the missing transverse energy distribution for the dilepton associated dark matter production in the SLHT model at the LHC for the 3 parameter sets of Table 5. The integrated luminosity is $300 \mathrm{fb}^{-1}$. 


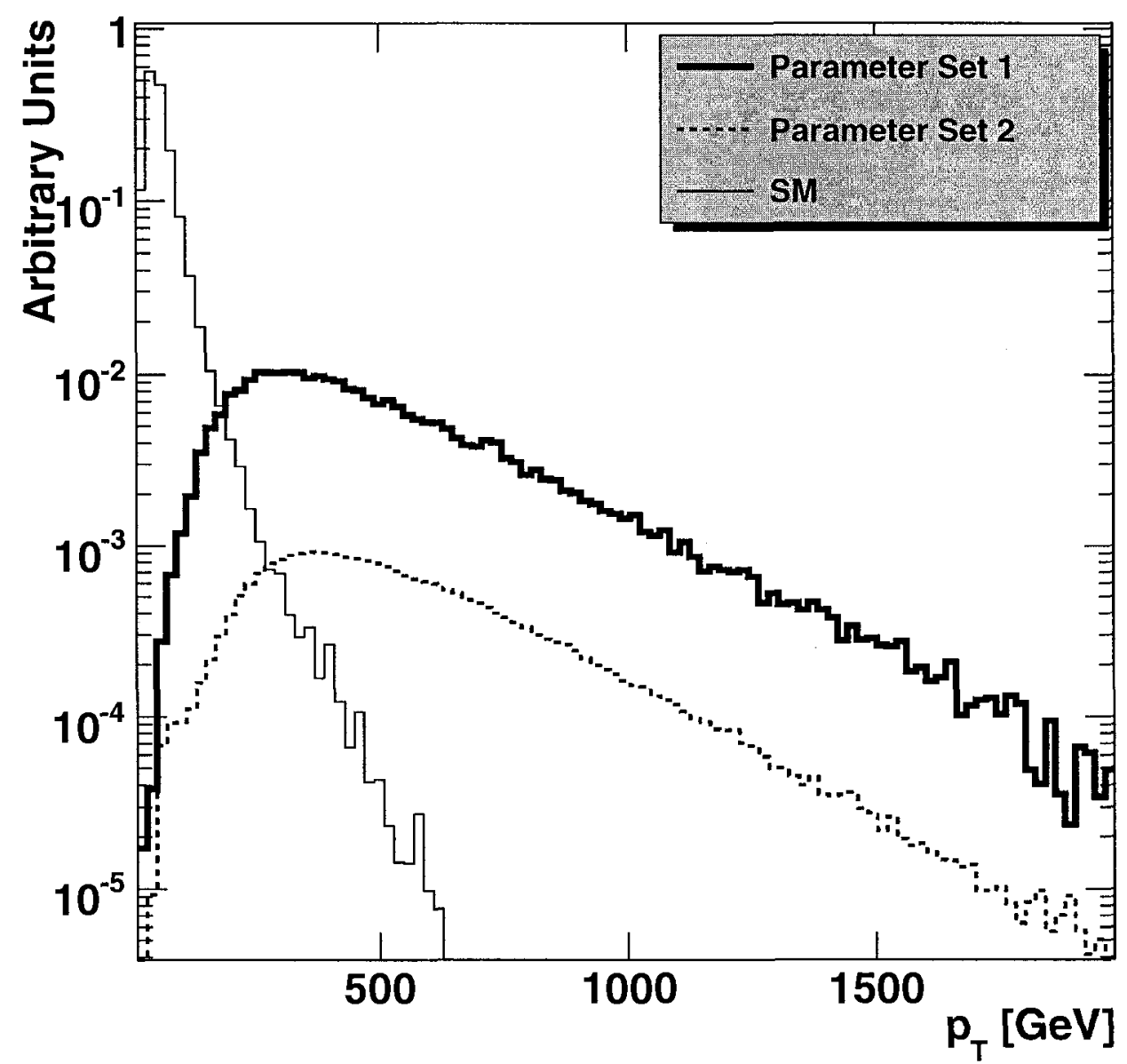

Figure 5.6: Expected $p_{T}$ distributions for the electrons in $p p \rightarrow l^{+} l^{-}+D M$ processes in the SLHT model for the first two parameter sets of Table 5. The SM $p_{T}$ distribution of electrons produced in $p p \rightarrow l^{+} l^{-}+$Missing Energy processes is also plotted for comparison. $l^{+} l^{-}$represents either $e^{+} e^{-}$or $\mu^{+} \mu^{-}$pair. All curves are normalized by their total cross sections. 


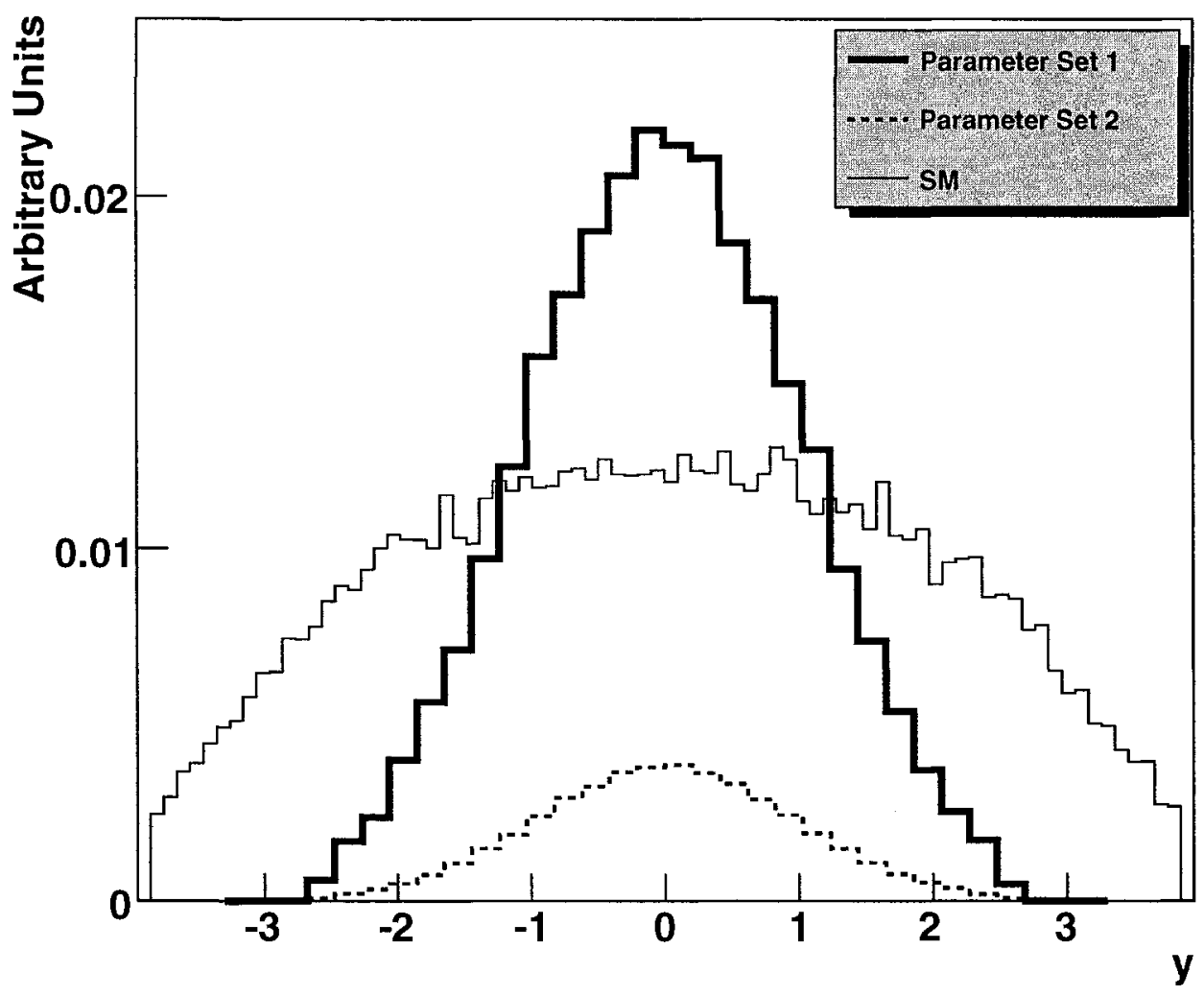

Figure 5.7: Rapidity distributions for the observed electrons in $p p \rightarrow l^{+} l^{-}+D M$ processes in the SLHT model for the first two parameter sets of Table 5. The SM model rapidity distribution of electrons produced in $p p \rightarrow l^{+} l^{-}+$Missing Energy processes is also presented. $l^{+} l^{-}$represents either $e^{+} e^{-}$or $\mu^{+} \mu^{-}$pair. All curves are normalized by their total cross sections. 


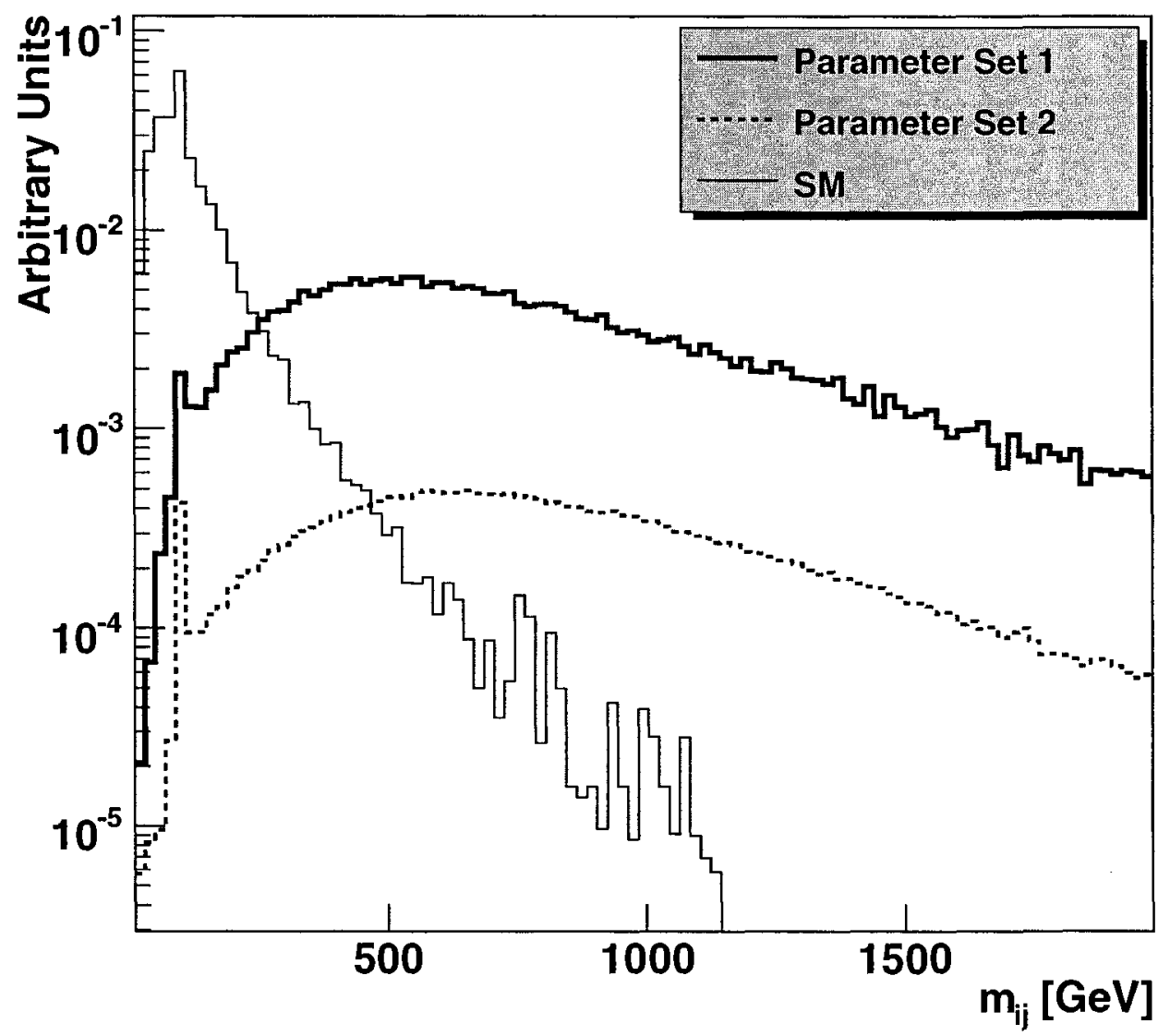

Figure 5.8: Invariant mass distributions of $e^{-}$and $e^{+}$in $p p \rightarrow l^{+} l^{-}+D M$ processes in the SLHT model for the first two parameter sets of Table 5. The SM model $m_{e^{+} e^{-}}$ distribution produced in $p p \rightarrow l^{+} l^{-}+$Missing Energy processes is also presented. $l^{+} l^{-}$represents either $e^{+} e^{-}$or $\mu^{+} \mu^{-}$pair. All curves are normalized by their total cross sections. 

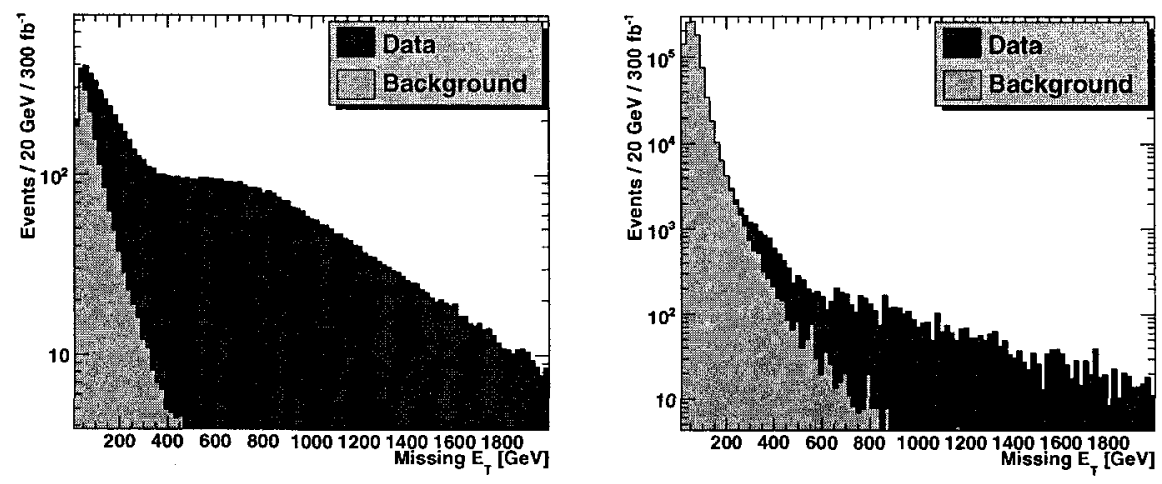

Figure 5.9: Missing transverse energy distribution in dilepton plus missing energy production for the parameter set 2 of Table 5 with $p_{T}>200 \mathrm{GeV}$ cut imposed(left) and without cuts (right). SM expectation for this channel is also presented.
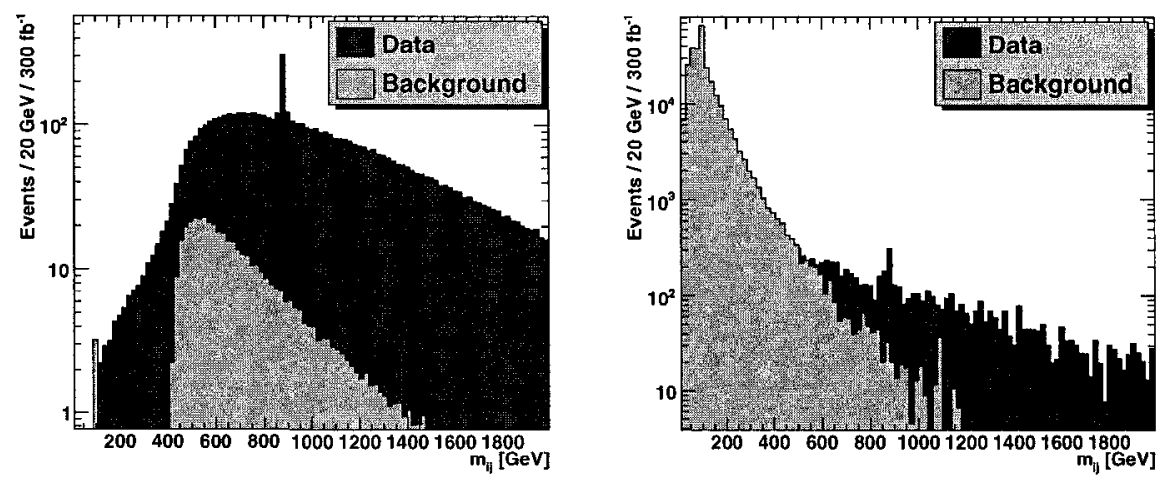

Figure 5.10: Invariant mass distribution of electrons in dilepton plus missing energy production for the second parameter set of Table 5 with $p_{T}>200 \mathrm{GeV}$ cut imposed(left) and without cuts (right). SM expectation for this channel is also presented. 


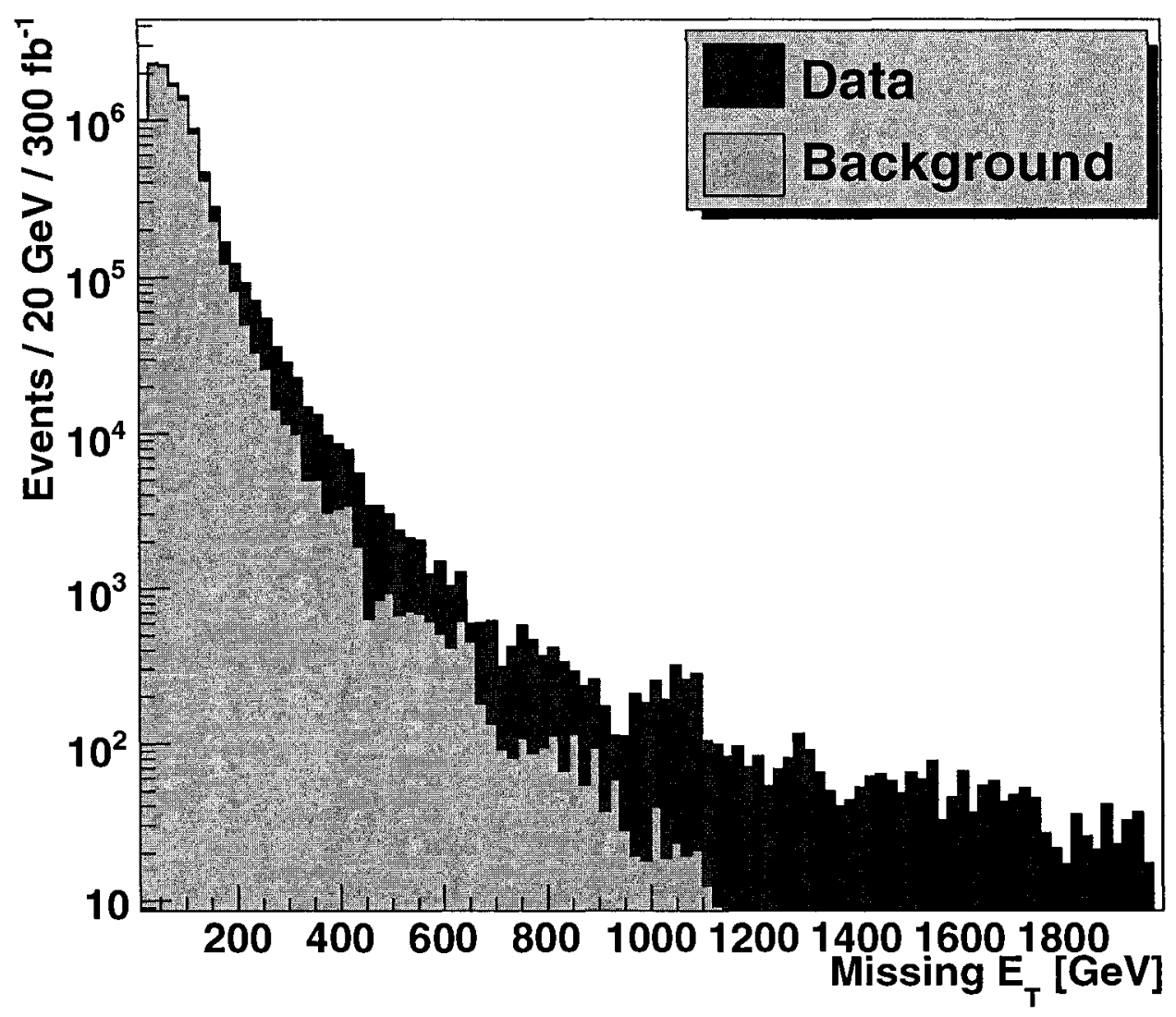

Figure 5.11: Expected missing transverse energy distribution for the $W^{ \pm}$associated dark matter production at the LHC in the Littlest Higgs with T-parity model for the dark matter mass $M_{A_{h}}=110 \mathrm{GeV}$ and symmetry breaking scale $f=700 \mathrm{GeV}$. The Higgs boson mass is chosen to be consistent with the relic density constraints of Eq. 2.8. This plot was generated using MadGraph for the integrated luminosity of $300 \mathrm{fb}^{-1}$. 


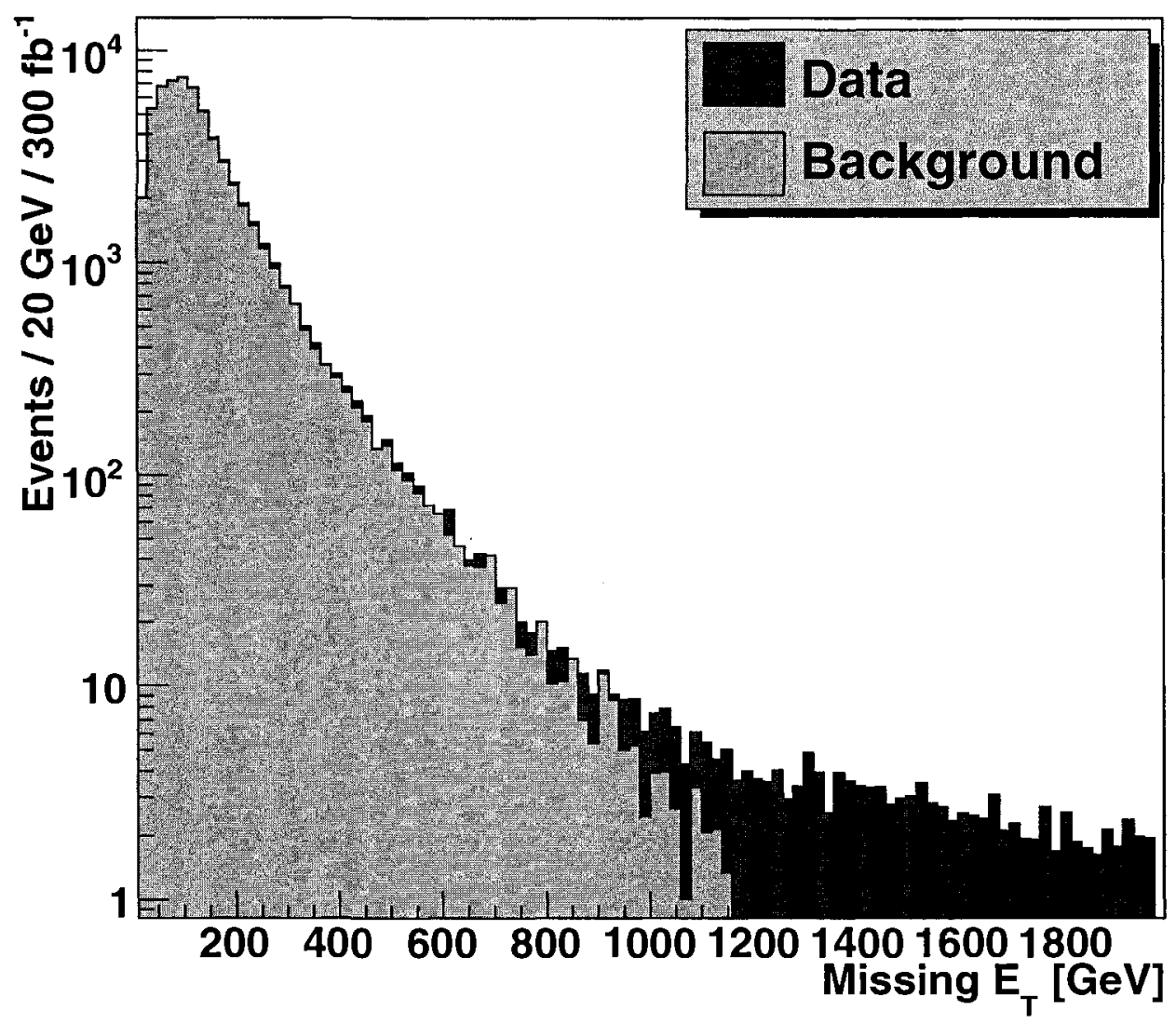

Figure 5.12: Missing transverse energy distribution for $p p \rightarrow A_{h} A_{h} W^{+} W^{-}$in the Littlest Higgs with T-parity model at the LHC and the SM background. This plot was generated with MadGraph using $M_{A_{h}}=110 \mathrm{GeV}$ and symmetry breaking scale $f=700 \mathrm{GeV}$, for the integrated luminosity of $300 \mathrm{fb}^{-1}$. 


\section{Chapter 6}

\section{Summary}

In this thesis, we have investigated dark matter candidates that arise in two Little Higgs models after the introduction of a discrete $Z_{2}$ symmetry called T-parity. We have discussed constraints that can be imposed on the parameter space of those models from astrophysical observations. A heavy neutrino is identified as a dark matter candidate in the Simplest Little Higgs with T-parity model and relic density constraints on that model's parameter space are imposed. It has been shown that, if the heavy neutrino is responsible for the observed dark matter in the universe, then its mass is linearly related to the value of the SLH symmetry breaking scale $f$ as $f \simeq 3.2 M_{N}+600$, where $f$ and $M_{N}$ are in $\mathrm{GeV}$.

We have analyzed couplings specific to the Simplest Little Higgs with T-parity (SLHT) model and introduced the model into the MadGraph package. Collider phenomenology of Little Higgs dark matter has been discussed and it has been shown that for a large region of the allowed parameter space dark matter particles could be detected at the LHC. Problems of the dark matter analysis at the LHC were outlined. We showed that SLHT has an important monojet plus missing energy signature that 
clearly distinguishes it from both the Littlest Higgs with T-parity model and from supersymmetry. A dileptonic channel of dark matter production was shown to be a promising independent channel of heavy neutrino production that can be used to confirm the SLHT mechanism if the monojet dark matter signal is detected. We also presented distributions of various kinematic functions for the leptons of the dilepton associated dark matter production and discussed kinematic cuts that provide significant enhancement of the signal to background ratio and thus permit observation of dark matter particles with higher mass. Dark matter signatures of the Littlest Higgs with T-parity model, which has been studied more extensively in the literature, were also discussed.

We also started a much broader investigation of the effects of dark matter microscopic properties on astrophysical observables and, in particular, looked at the impact of kinetic decoupling on structure formation. We discussed modifications to the power spectrum of cosmological perturbations that arise when the kinetic coupling of dark matter to the primordial plasma is taken into account. Modification of the transfer function of the dark matter perturbations has been shown and the rate coefficient that determines the time of kinetic decoupling has been derived for the case of the Littlest Higgs with T-parity model. 


\section{A Feynman Rules for the Simplest Little Higgs with T-parity model}

In this appendix we provide a list of Feynman rules specific to the SLHT model. As discussed in Section 2.1 implementing T-parity in the gauge and scalar sectors of the Simplest Little Higgs model does not require significant changes to the structure of the original Simplest Little Higgs model. The major effect in the gauge sector is to set the aligned vevs of the two global $\left[S U(3)_{W} \otimes U(1)_{X}\right]_{i}$ symmetry factors equal, $f_{1}=f_{2}$. In the notation of [50] this requirement leads to $\tan \beta=f_{2} / f_{1}=1$. We summarize the triple and quartic gauge boson vertex factors in Tables A1 and A2, respectively. The triple and quartic couplings are given in the form

$$
\begin{gathered}
V_{1}^{\mu}\left(k_{1}\right) V_{2}^{\nu}\left(k_{2}\right) V_{3}^{\rho}\left(k_{3}\right): \quad-i g_{V_{1} V_{2} V_{3}}\left[g^{\mu \nu}\left(k_{1}-k_{2}\right)^{\rho}+g^{\nu \rho}\left(k_{2}-k_{3}\right)^{\mu}+g^{\rho \mu}\left(k_{3}-k_{1}\right)^{\nu}\right] \\
V_{1}^{\mu} V_{2}^{\nu} V_{3}^{\rho} V_{4}^{\sigma}: \quad-i g_{V_{1} V_{2} V_{3} V_{4}}\left(2 g^{\mu \nu} g^{\rho \sigma}-g^{\mu \rho} g^{\nu \sigma}-g^{\nu \rho} g^{\mu \sigma}\right)
\end{gathered}
$$

where $g_{V_{1} V_{2} V_{3}}$ and $g_{V_{1} V_{2} V_{3} V_{4}}$ are vertex factors provided in the tables. We also must note that the $W^{-} Z^{0} X^{+} Y^{0}$ and $W^{+} Z^{0} X^{-} \bar{Y}^{0}$ couplings can not be written in the standard form due to the approximate nature of the gauge eigenstates. The appropriate couplings have the form:

$$
\begin{aligned}
& W_{\mu}^{-} Z_{\nu}^{0} X_{\rho}^{+} Y_{\sigma}^{0}: \frac{-i s_{W}^{2}}{2 \sqrt{2} c_{W}}\left[2 g_{\mu \nu} g_{\rho \sigma}-g_{\mu \sigma} g_{\nu \rho}-g_{\mu \rho} g_{\nu \sigma}\right]-3 c_{W}^{2}\left(g_{\mu \sigma} g_{\nu \rho}-g_{\mu \rho} g_{\nu \sigma}\right) \\
& W_{\mu}^{+} Z_{\nu}^{0} X_{\rho}^{-} \bar{Y}_{\sigma}^{0}: \frac{-i s_{W}^{2}}{2 \sqrt{2} c_{W}}\left[2 g_{\mu \nu} g_{\rho \sigma}-g_{\mu \sigma} g_{\nu \rho}-g_{\mu \rho} g_{\nu \sigma}\right]-3 c_{W}^{2}\left(g_{\mu \sigma} g_{\nu \rho}-g_{\mu \rho} g_{\nu \sigma}\right) .
\end{aligned}
$$

The fermion sector of the Simplest Little Higgs with T-parity model is very different from the original Simplest Little Higgs. The modifications are especially significant in the Yukawa interactions. Things are also complicated by the fact that, 


\begin{tabular}{|c|c||c|c|}
\hline Particles & $g_{V V V}$ & Particles & $g_{V V V}$ \\
\hline$W^{+} W^{-} A$ & $-g s_{W}$ & $X^{+} X^{-} A$ & $-g s_{W}$ \\
\hline$W^{+} W^{-} Z^{0}$ & $-g c_{W}$ & $X^{+} X^{-} Z^{0}$ & $-\frac{g}{2} \frac{1-2 s_{W}^{2}}{c_{W}}$ \\
\hline$W^{+} W^{-} Z_{h}$ & 0 & $X^{+} X^{-} Z_{h}$ & $\frac{g}{2} \sqrt{3-t_{W}^{2}}$ \\
\hline$Y^{0} \bar{Y}^{0} Z^{0}$ & $-\frac{1}{2 c_{W}} g$ & $Y^{0} \bar{Y}^{0} A$ & 0 \\
\hline$Y^{0} \bar{Y}^{0} Z_{h}$ & $-\frac{g}{2} \sqrt{3-t_{W}^{2}}$ & $X^{+} W^{-} Y^{0}$ & $-\frac{g}{\sqrt{2}}$ \\
\hline
\end{tabular}

Table A1: Feynman rules for the triple gauge boson interactions in the Simplest Little Higgs with T-parity model. All momenta are defined as outgoing. The SM couplings of $W^{ \pm}$to $Z^{0}$ and photon are also presented for reference. In the table $s_{W}, c_{W}$, and $t_{W}$ stand for the sine, cosine and tangent of the electroweak mixing angle respectively.

choosing the anomaly-free implementation of fermion assignments, the third generation quarks transform differently from the quarks of the first two generations under the global symmetry of the model. For the purposes of dark matter study we ignore mixings in the leptonic and quark sectors. This leads to a considerable simplification in the Feynman rules, and allows us to concentrate on the most significant "smokinggun" signatures of the model. The more complete set of couplings including $v^{2} / f^{2}$ corrections and various mixing can be derived from generic Simplest Little Higgs Lagrangians provided in [45] after imposing the T-parity requirements described in Section 2.2. In Table A3 we show scalar-fermion couplings that arise from the Yukawa Lagrangians introduced in Section 2.2.

The fermion couplings to the gauge bosons are determined by the standard fermion kinetic term:

$$
\mathcal{L}=\bar{\psi} i \mathcal{D}_{\mu} \gamma^{\mu} \psi, \quad \mathcal{D}=\partial+i g A^{a} T^{a}+i g_{x} Q_{x} B^{x}
$$




\begin{tabular}{|c|c||c|c|}
\hline Particles & $g_{V V V V}$ & Particles & $g_{V V V V}$ \\
\hline$W^{+} W^{-} A A$ & $g^{2} s_{W}^{2}$ & $W^{+} W^{+} W^{-} W^{-}$ & $-g^{2}$ \\
\hline$X^{+} X^{-} A A$ & $g^{2} s_{W}^{2}$ & $X^{+} X^{-} Z^{0} Z^{0}$ & $\frac{g^{2}}{4 c_{W}^{2}}\left(1-2 s_{W}^{2}\right)^{2}$ \\
\hline$X^{+} X^{-} Z_{h} Z_{h}$ & $\frac{g^{2}}{4}\left(3-t_{W}^{2}\right)$ & $X^{+} X^{-} A Z^{0}$ & $\frac{g^{2} s_{W}}{2 c_{W}}\left(1-2 s_{W}^{2}\right)$ \\
\hline$X^{+} X^{-} A Z_{h}$ & $-\frac{g^{2} s_{W}}{2} \sqrt{3-t_{W}^{2}}$ & $X^{+} X^{-} Z^{0} Z_{h}$ & $-\frac{g^{2}}{4 c_{W}}\left(1-2 s_{W}^{2}\right) \sqrt{3-t_{W}^{2}}$ \\
\hline$Y^{0} \bar{Y}^{0} Z_{h} Z_{h}$ & $\frac{g^{2}}{4}\left(3-t_{W}^{2}\right)$ & $Y^{0} \bar{Y}^{0} Z^{0} Z^{0}$ & $\frac{1}{4 c_{W}^{2}} g^{2}$ \\
\hline$Y^{0} \bar{Y}^{0} Z_{h} Z^{0}$ & $\frac{g^{2}}{4 c_{W}} \sqrt{3-t_{W}^{2}}$ & $Y^{0} \bar{Y}^{0} Y^{0} \bar{Y}^{0}$ & $g^{2}$ \\
\hline$W^{-} X^{+} A Y^{0}$ & $\frac{s_{W}}{\sqrt{2}} g^{2}$ & $W^{-} Z_{h} X^{+} Y^{0}$ & $\frac{g^{2}}{2 \sqrt{2}} \sqrt{3-t_{W}^{2}}$ \\
\hline$W^{+} X^{-} A \bar{Y}^{0}$ & $\frac{s_{W}}{\sqrt{2}} g^{2}$ & $W^{+} Z_{h} X^{-} \bar{Y}^{0}$ & $\frac{g^{2}}{2 \sqrt{2}} \sqrt{3-t_{W}^{2}}$ \\
\hline$X^{+} \bar{Y}^{0} X^{-} Y^{0}$ & $-\frac{g^{2}}{2}$ & $X^{+} X^{-} X^{+} X^{-}$ & $g^{2}$ \\
\hline$W^{+} Y^{0} W^{-} \bar{Y}^{0}$ & $-\frac{g^{2}}{2}$ & $W^{+} X^{+} W^{-} X^{-}$ & $-\frac{g^{2}}{2}$ \\
\hline
\end{tabular}

Table A2: Feynman rules for the quartic gauge boson interactions in the Simplest Little Higgs with T-parity model. Sample SM couplings are also provided for reference.

\begin{tabular}{|c|c||c|c||c|c|}
\hline Particles & Vertices & Particles & Vertices & Particles & Vertices \\
\hline$\eta \bar{N}_{m} \nu_{m}$ & $-i \frac{v M_{N_{m}}}{2 f^{2}} P_{L}$ & $\eta \bar{T} t$ & $-i \frac{M_{T}}{\sqrt{2} f} P_{L}$ & $\eta \bar{D} d$ & $-i \frac{v M_{D}}{2 f^{2}} P_{L}$ \\
\hline$\eta \eta \bar{N}_{m} N_{m}$ & $\frac{M_{N_{m}}}{2 f^{2}}$ & $\eta \eta \bar{T} T$ & $\frac{M_{T}}{2 f^{2}}$ & $\eta \eta \bar{D} D$ & $\frac{M_{D}}{2 f^{2}}$ \\
\hline$h \bar{N}_{m} N_{m}$ & $\frac{v M_{N_{m}}}{2 f^{2}}$ & $h \bar{T} T$ & $\frac{v M_{T}}{2 f^{2}}$ & $h \bar{D} D$ & $\frac{v M_{D}}{2 f^{2}}$ \\
\hline$h h \bar{N}_{m} N_{m}$ & $\frac{M_{N_{m}}}{2 f^{2}}$ & $h h \bar{T} T$ & $\frac{M_{T}}{2 f^{2}}$ & $h h \bar{D} D$ & $\frac{M_{D}}{2 f^{2}}$ \\
\hline
\end{tabular}

Table A3: Feynman rules for scalar-fermion couplings in the Simplest Little Higgs with T-parity model. $P_{L}=\left(1-\gamma^{5}\right) / 2$ is a projection operator. Only the leading terms are provided. 
with the $Q_{x}$ charges given in Table 2.1. $T^{a}$ are the generators of the fundamental 3 representation of the $S U(3)_{W}$. In the case of the first and second generation quarks $T^{a}$ should be replaced by $-T^{a *}$ - generators of the antifundamental $\overline{\mathbf{3}}$ representation of the $S U(3)_{W}$. Fermion couplings to the gauge bosons in the SLHT are identical to those of the original Simplest Little Higgs model [50] and are provided in Table A4. We note, however, that our value for the coupling of $D$ and $S$ quarks to the heavy $Z_{h}$ is slightly different from the coupling calculated in [50].

Finally, in Table A5, we provide couplings of the new heavy gauge bosons and the scalar Higgs boson. We also note that, because the new electroweak singlet $\eta$ is a real scalar field, it does not couple directly to the gauge bosons, while its quartic couplings are $f^{2}$ suppressed. Therefore, for the investigation of dark matter phenomenology, effects of the new heavy scalar are completely negligible. 


\begin{tabular}{|c|c|}
\hline Particles & Vertices \\
\hline$Z^{0 \mu} \bar{e} e:$ & $\frac{g}{2 c_{W}} \gamma^{\mu}\left[\left(c_{W}^{2}-s_{W}^{2}\right) P_{L}-2 s_{W}^{2} P_{R}\right]$ \\
\hline$Z_{h}^{\mu} \bar{t} t:$ & $-\frac{g}{c_{W} \sqrt{3-4 s_{W}^{2}}} \gamma^{\mu}\left[\left(\frac{1}{2}-\frac{1}{3} s_{W}^{2}\right) P_{L}+\frac{2}{3} s_{W}^{2} P_{R}\right]$ \\
\hline$Z_{h}^{\mu} \bar{b} b:$ & $-\frac{g}{c_{W} \sqrt{3-4 s_{W}^{2}}} \gamma^{\mu}\left[\left(\frac{1}{2}-\frac{1}{3} s_{W}^{2}\right) P_{L}-\frac{1}{3} s_{W}^{2} P_{R}\right]$ \\
\hline$Z_{h}^{\mu} \bar{u} u:$ & $-\frac{g}{c_{W} \sqrt{3-4 s_{W}^{2}}} \gamma^{\mu}\left[\left(-\frac{1}{2}+\frac{2}{3} s_{W}^{2}\right) P_{L}+\frac{2}{3} s_{W}^{2} P_{R}\right]$ \\
\hline$Z_{h}^{\mu} \bar{d} d:$ & $-\frac{g}{c_{W} \sqrt{3-4 s_{W}^{2}}} \gamma^{\mu}\left[\left(-\frac{1}{2}+\frac{2}{3} s_{W}^{2}\right) P_{L}-\frac{1}{3} s_{W}^{2} P_{R}\right]$ \\
\hline$Z_{h}^{\mu} \bar{e} e:$ & $-\frac{g}{c_{W} \sqrt{3-4 s_{W}^{2}}} \gamma^{\mu}\left[\left(\frac{1}{2}-s_{W}^{2}\right) P_{L}-s_{W}^{2} P_{R}\right]$ \\
\hline$Z_{h}^{\mu} \bar{\nu} \nu:$ & $-\frac{g}{c_{W} \sqrt{3-4 s_{W}^{2}}} \gamma^{\mu}\left(\frac{1}{2}-s_{W}^{2}\right) P_{L}$ \\
\hline$Z_{h}^{\mu} \bar{T} T:$ & $-\frac{g}{c_{W} \sqrt{3-4 s_{W}^{2}}} \gamma^{\mu}\left[\left(-1+\frac{5}{3} s_{W}^{2}\right) P_{L}+\frac{2}{3} s_{W}^{2} P_{R}\right]$ \\
\hline$Z_{h}^{\mu} \bar{D} D:$ & $-\frac{g}{c_{W} \sqrt{3-4 s_{W}^{2}}} \gamma^{\mu}\left[\left(1-\frac{4}{3} s_{W}^{2}\right) P_{L}-\frac{1}{3} s_{W}^{2} P_{R}\right]$ \\
\hline$Z_{h}^{\mu} \bar{N} N:$ & $-\frac{g}{c_{W} \sqrt{3-4 s_{W}^{2}}} \gamma^{\mu}\left(-1+s_{W}^{2}\right) P_{L}$ \\
\hline$X_{\mu}^{-} \bar{D} u:$ & $\frac{i g}{\sqrt{2}} \gamma_{\mu} P_{L}$ \\
\hline$Y_{\mu}^{0} \bar{D} d:$ & $-\frac{i g}{\sqrt{2}} \gamma_{\mu} P_{L}$ \\
\hline$X_{\mu}^{-\bar{e} N}:$ & $-\frac{i g}{\sqrt{2}} \gamma_{\mu} P_{L}$ \\
\hline$Y_{\mu}^{0} \bar{\nu} N:$ & $-\frac{i g}{\sqrt{2}} \gamma_{\mu} P_{L}$ \\
\hline$X_{\mu}^{-} \bar{b} T:$ & $-\frac{i g}{\sqrt{2}} \gamma_{\mu} P_{L}$ \\
\hline$Y_{\mu}^{0} \bar{t} T:$ & $-\frac{i g}{\sqrt{2}} \gamma_{\mu} P_{L}$ \\
\hline
\end{tabular}

Table A4: Feynman rules for gauge boson-fermion couplings in the Simplest Little Higgs with T-parity model. $P_{L}=\left(1-\gamma^{5}\right) / 2$ and $P_{R}=\left(1+\gamma^{5}\right) / 2$ are projection operators. Couplings for the second generation quarks are identical to those of the first generation. The SM lepton $Z^{0}$-boson coupling is also provided to clarify our phase choice. Only the leading terms are provided. 


\begin{tabular}{|c|c|}
\hline Particles & Vertices \\
\hline$W^{+} W^{-} h:$ & $\frac{1}{2} g^{2} v$ \\
\hline$X^{+} X^{-} h:$ & $-\frac{1}{2} g^{2} v$ \\
\hline$X^{+} X^{-} h h:$ & $-\frac{1}{2} g^{2}$ \\
\hline$Y^{0} \bar{Y}^{0} h:$ & $\frac{g^{2} v}{8} v^{2}$ \\
\hline$Y^{0} \bar{Y}^{0} h h:$ & $\frac{g^{2}}{8} v^{2}$ \\
\hline$Z_{h} Z_{h} h:$ & $-\frac{g^{2} v}{2 c_{W}^{2}}$ \\
\hline$Z_{h} Z_{h} h h:$ & $-\frac{g^{2}}{2 c_{W}^{2}}$ \\
\hline$Z^{0} Z_{h} h:$ & $\frac{1}{2} g^{2} v \frac{\left(1-t_{W}^{2}\right)}{c_{W} \sqrt{3-t_{W}^{2}}}$ \\
\hline$Z^{0} Z_{h} h h:$ & $\frac{1}{2} g^{2} \frac{\left(1-t_{W}^{2}\right)}{c_{W} \sqrt{3-t_{W}^{2}}}$ \\
\hline
\end{tabular}

Table A5: Feynman rules for gauge boson-scalar couplings in the Simplest Little Higgs with T-parity model. The SM coupling of the $W^{ \pm}$to the Higgs field $h$ is also provided for reference. Factor of $g_{\mu \nu}$ is implied in each vertex.

\section{B Parameters of the Simplest Little Higgs with T-parity model}

In this appendix we discuss calculation methods for the parameters of the Simplest Little Higgs with T-parity model that are used in various parts of this thesis. First of all we need to emphasize that, after the decoupling of additional heavy fermions only three independent parameters are introduced in the model: $f$ - the energy scale of the initial $S U(3)_{c} \otimes S U(3)_{W} \otimes U(1)_{X}$ global symmetry, $M_{h}$ - the mass of the Higgs boson and $M_{N}$ - the mass of the lightest T-odd particle. The mass of the Higgs boson does not have a significant effect on either the collider signatures or the relic density 
calculations of the SLHT dark matter and we fix its value as an input parameter $M_{h}$ $=120 \mathrm{GeV}$.

For the calculation of cross sections and simulation of collider events with MadGraph we are required to introduce masses and widths of new particles into the simulation package. Masses of the new gauge bosons in the leading order depend only on the symmetry breaking scale $f$, while fermion masses depend also on the Yukawa couplings.

$$
M_{Z_{h}}=\frac{\sqrt{2} g f}{\sqrt{3-t_{W}^{2}}}, \quad M_{X^{ \pm}}=M_{Y^{0}}=\frac{g f}{\sqrt{2}}, \quad M_{f}=\sqrt{2} \lambda_{f} f
$$

In this research we assume a universal value of the Yukawa couplings $\lambda_{f}=1 / 2$ for all new fermions with the exception of the first generation heavy neutrino. This choice provides heavy fermion masses large enough so that they don't have a significant influence on the dark matter phenomenology, but still small enough not to generate the naturalness problem in the Higgs mass parameter.

The two-body decay width of the new heavy particles can be written as

$$
\Gamma=\frac{S|\mathcal{M}|^{2} p_{0}}{8 \pi m_{1}^{2}}
$$

where $\mathrm{S}$ is a statistical factor $1 / j$ ! for each group of $j$ identical particles in the final state, $m_{1}$ - mass of a decaying particle, $|\mathcal{M}|$ - invariant amplitude of a decay process, and $p_{0}$ - invariant momentum, given by [71]

$$
p_{0}=\frac{1}{2 m_{1}} \sqrt{m_{1}^{4}+m_{2}^{4}+m_{3}^{4}-2 m_{1}^{2} m_{2}^{2}-2 m_{1}^{2} m_{3}^{2}-2 m_{2}^{2} m_{3}^{2}} .
$$

For the calculation of the decay widths we wrote a Mathematica [69] code with the invariant decay amplitudes calculated using the Feyncalc package [70]. For instance, we can write the following invariant amplitudes averaged over initial and summed 
over final spin states for the $Z_{h} \rightarrow \bar{l}$ and $Z_{h} \rightarrow Z^{0} h$ decays:

$$
\begin{gathered}
\left|\mathcal{M}\left(Z_{h} \rightarrow l \bar{l}\right)\right|^{2}=\frac{2}{3}\left[\frac{g}{2 c_{W} \sqrt{3-4 s_{W}^{2}}}\right]^{2}\left(M_{Z_{h}}^{2}\left(1-4 s_{W}^{2}+8 s_{W}^{4}\right)-M_{l}^{2}\left(1+8 s_{W}^{2}-16 s_{W}^{4}\right)\right) \\
\left|\mathcal{M}\left(Z_{h} \rightarrow Z^{0} h\right)\right|^{2}=\frac{1}{3}\left[\frac{g^{2} v c_{W}}{2 \sqrt{3-t_{W}^{2}}}\left(1-t_{W}^{4}\right)\right]^{2}\left(3+\frac{p_{0}^{2}}{M_{Z^{0}}^{2}}\right) .
\end{gathered}
$$

In Fig. B1 we show an example of the branching ratios of the $Z_{h}$ boson decay into $N \bar{N}, q \bar{q}, \nu \bar{\nu}, \bar{l}$ and $Z^{0} h$ for $f=1560 \mathrm{GeV}, M_{Z_{h}}=872 \mathrm{GeV}, M_{h}=120 \mathrm{GeV}$, and $M_{N}=300 \mathrm{GeV}$.

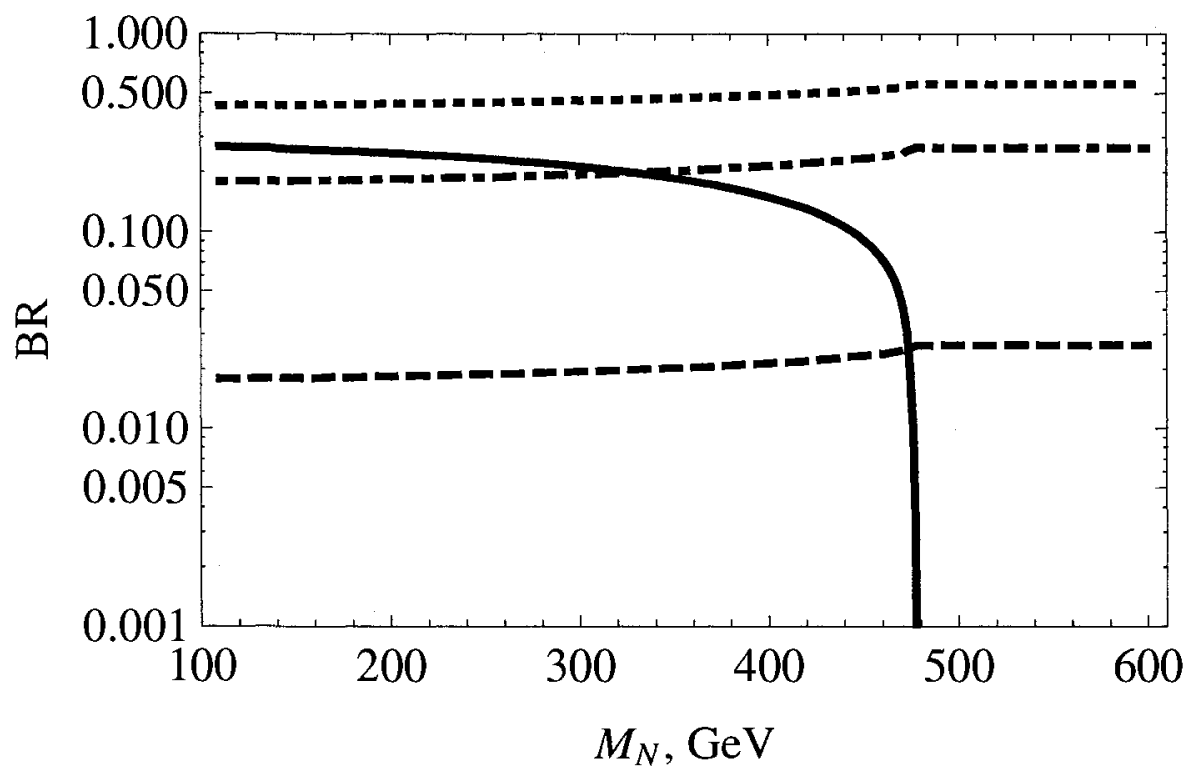

Figure B1: $Z_{h}$ decay branching ratios in the Simplest Little Higgs with T-parity model for $f=1560 \mathrm{GeV}, M_{Z_{h}}=872 \mathrm{GeV}$, and $M_{h}=120 \mathrm{GeV}$. The solid, dotted, dashed and dash-dotted lines are the contributions of the dark matter, quark, neutrino, and $Z_{h} \rightarrow Z^{0}+h$ decay channels respectively. The total decay width of the heavy $Z_{h}$ boson is $\Gamma=5.8 \mathrm{GeV}$. 


\section{Bibliography}

[1] S. Glashow, Nucl. Phys. 22, 579 (1961); S. Weinberg, Phys. Rev. Lett. 19, 1264 (1967); A. Salam, In proceedings of the 8th Nobel Sympothium, Stokholm, 1968, edited by N. Svartholm (Almquist and Wiksells, Stokholm, 1968), p.367.

[2] D.N.Spergel et. al, ApJS 170, 377 (2007) [astro-ph/0603450].

[3] M. Tegmark et. al, Phys. Rev. D 69, 103501 (2004) [astro-ph/0310723].

[4] G.R. Blumenthal et. al, Nature 311, 517 (1984).

[5] J. Sellwood, A. Kosowsky, "The Dynamics, Structure and History of Galaxies" 273, 243 (2002) [astro-ph/0109555].

[6] N. Arkani-Hamed, A. G. Cohen and H. Georgi, Phys. Lett. B 513, 232 (2001) [hep-ph/0105239].

[7] N. Arkani-Hamed, A. G. Cohen, T. Gregoire and J. G. Wacker, JHEP 0208, 020 (2002) [hep-ph/0202089].

[8] N. Arkani-Hamed, A. G. Cohen, E. Katz, A. E. Nelson, T. Gregoire and J. G. Wacker, JHEP 0208, 021 (2002) [hep-ph/0206020].

[9] N. Arkani-Hamed, A. G. Cohen, E. Katz and A. E. Nelson, JHEP 0207, 034 (2002) [hep-ph/0206021]. 
[10] I. Low, W. Skiba and D. Smith, Phys. Rev. D 66, 072001 (2002) [hep-ph/0207243].

[11] For a recent review, see e. g., M. Schmaltz, hep-ph/0210415.

[12] A. Martin, [hep-ph/0602206].

[13] H. C. Cheng and I. Low, JHEP 0309, 051 (2003) [hep-ph/0308199].

[14] I. Low, JHEP 0410, 067 (2004) [hep-ph/0409025].

[15] H. C. Cheng and I. Low, JHEP 0408, 061 (2004) [hep-ph/0405243].

[16] J. Hubisz and P. Meade, Phys. Rev. D 71, 035016 (2005) [hep-ph/0411264].

[17] D. Spergel, P. Steinhardt, Phys. Rev. Lett. 84, 3760 (2000) [astro-ph/9909386]

[18] P. Bode, J. Ostriker, N. Turok, ApJ. 556, 93 (2001) [astro-ph/0010389].

[19] E. Bertschinger, Phys. Rev. D 74, 063509 (2006) [astro-ph/0607319].

[20] A. Loeb, M. Zaldarriaga, Phys.Rev.D 71, 103520 (2005) [astro-ph/0504112].

[21] S. Novaes, in "Particle and Fields", Proceedings of the 10th J.A. Swieca Summer School, San Paulo, Brazil, 1999 (World Scientific, Singapore, 2000) [hep-ph/0001283].

[22] P. Higgs, Phys. Lett. 12, 132 (1964); Phys. Rev. Lett. 13, 508 (1964); Phys. Rept. 145, 1156 (1966); F. Englert and R. Brout, Phys. Rev. Lett. 13, 321 (1964); G. Guralnik, C. Hagen, and T. Kibble, Phys. Rev. Lett. 13, 585 (1964); T. Kibble, Phys. Rep. 155, $1554(1967)$.

[23] A. Djouadi, Phys. Rept. 457, 001 (2005) [arXiv:hep-ph/0503172].

[24] Y. Ashie et. al, Phys. Rev. Lett. 93, 101801 (2004) [hep-ex/0404034].

[25] B. Aharmim et. al (SNO Collaboration), Complete (archival) version of SNO Phase I results (2006) [nucl-ex/0610020]. 
[26] K. G. Begeman, A. H. Broeils and R. H. Sanders, MNRAS 249, 523 (1991).

[27] R. Massey et. al, Nature 445, 286 (2007) [astro-ph/0701594].

[28] M. Sun et. al, [astr0-ph/0805.2320].

[29] D. Clowe et. al, ApJ 648, L109 (2006).

[30] V. Springel and G. Farrar, [astro-ph/0703232].

[31] M. Tegmark, M. Zaldarriaga and A. Hamilton, Phys.Rev. D 63, 043007 (2001) [astro-ph/0008167].

[32] V. Springel, C. Frenk and S. White, Nature, 440, 1137 (2006) [astro-ph/0604561].

[33] E. Kolb and M. Turner, The Early Universe, (1994).

[34] S. Dodelson, Modern Cosmology, (2003).

[35] G. Jungman, M. Kamionkowski, K. Griest, Phys. Rep. 267, 195 (1996).

[36] M. Srednicki, R. Watkins and K. Olive, Nucl. Phys. B 310, 693 (1988).

[37] L. Roszkowski, Phys. Rev. D 50, 4842 (1994) [hep-ph/9404227].

[38] G. Bertone, D. Hooper and J. Silk, Phys. Rept. 405, 297 (2005) [hep-ph/0404175].

[39] W.-M. Yao et. al, J. Phys. G 33, 1 (2006).

[40] K. Griest and D. Seckel, Phys. Rev. D 43, 3191 (1991).

[41] D. E. Kaplan and M. Schmaltz, JHEP 0310, 039 (2003) [hep-ph/0302049].

[42] M. Schmaltz, JHEP 0408, 056 (2004) [hep-ph/0407143].

[43] C. Hill and R. Hill, Phys. Rev. D 76, 115014 (2007) [hep-ph/0705.0697].

[44] C. Csaki et. al, [hep-ph/0804.0622]. 
[45] T. Han et. al, Phys. Rev. D 67, 095004 (2003) [hep-ph/0301040].

[46] Q. Cao and C. Chen, [hep-ph/0707.0877].

[47] A. Birkedal et. al, Phys.Rev. D bf 74, 035002 (2006) [hep-ph/0603077].

[48] C. Chen, K. Cheung and T. Yuan, Phys.Lett. B 644, 158 (2007) [hep-ph/0605314].

[49] A. Belyaev et. al, Phys.Rev. D 74, 115020 (2006) [hep-ph/0609179].

[50] T. Han, H. Logan and L. Wang, JHEP 0601, 099 (2006) [hep-ph/0506313].

[51] X. Chen, M. Kamionkowski and X. Zhang, Phys. Rev. D 64, 021302 (2001) [astro-ph/0103452].

[52] A. Green, S. Hofmann and D. Schwarz, JCAP 08, 003 (2005) [astro-ph/0503387].

[53] M. Kuhlen, J. Diemand and P. Madau, [astro-ph/0805.4416].

[54] E. Siegel, M. Hertzberg and J. Fry, [astro-ph/0702546].

[55] H. Baer and X. Tata, [hep-ph/0805.1905].

[56] Haifeng Pi et. at, CMS NOTE, 035, (2006).

[57] Atlas Technical Design Report V1, (1999).

[58] J. Alwall et. al, JHEP 0709, 028 (2007) [hep-ph/0706.2334].

[59] V. Barger and R. Phillips, Collider Physics, 1997.

[60] J. Pumplin et. al, JHEP, 0602, 032 (2006) [hep-ph/0512167].

[61] J. Hubisz, A. Noble and M. Perelstein, http://www.lepp.cornell.edu/maxim/LHTtool/

[62] H. Murayama, I. Watanabe, and K. Hagiwara, HELAS: HELicity amplitude subroutines for Feynman diagram evaluations, KEK-91-11. 
[63] T. Sjostrand, S. Mrenna, and P. Skands, JHEP 05, 026 (2006) [hep-ph/0603175].

[64] TopDrawer, available at http://www-group.slac.stanford.edu/beamphysics/codes/topdrawer/.

[65] E. Boos et. al, Nucl. Instrum. Meth. A534, 250 (2004) [hep-ph/0403113].

[66] A. Pukhov, [hep-ph/0412191].

[67] T. Gleisberg et. al, JHEP, 02, 056 (2004) [hep-ph/0311263].

[68] W. Kilian, T. Ohl and J. Reuter, [hep-ph/0708.4233].

[69] Wolfram Research, Inc., Mathematica 6.0, (2007) http://www.wolfram.com/.

[70] R. Mertig, M. Bohm and A. Denner, Comp. Phys. Comm. 64, 345 (1991).

[71] D. Griffiths, Introduction to elementary particles, (1987). 